\title{
Ultracold atomic Fermi-Bose mixtures in bichromatic optical dipole traps: a novel route to study fermion superfluidity
}

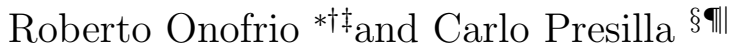

November 8, 2018

\begin{abstract}
The study of low density, ultracold atomic Fermi gases is a promising avenue to understand fermion superfluidity from first principles. One technique currently used to bring Fermi gases in the degenerate regime is sympathetic cooling through a reservoir made of an ultracold Bose gas. We discuss a proposal for trapping and cooling of two-species Fermi-Bose mixtures into optical dipole traps made from combinations of laser beams having two different wavelengths. In these bichromatic traps it is possible, by a proper choice of the relative laser powers, to selectively trap the two species in such a way that fermions experience a stronger confinement than bosons. As a consequence, a deep Fermi degeneracy can be reached having at the same time a softer degenerate regime for the Bose gas. This leads to an increase in the sympathetic cooling efficiency and allows for higher precision thermometry of the Fermi-Bose mixture.
\end{abstract}

KEY WORDS: Bose and Fermi degenerate gases; superfluidity and superconductivity; evaporative cooling; sympathetic cooling; nonequilibrium statistical mechanics; Bose-Fermi mixtures.

\footnotetext{
*Dipartimento di Fisica "G. Galilei", Università di Padova, Via Marzolo 8, Padova 35131, Italy

${ }^{\dagger}$ Istituto Nazionale per la Fisica della Materia, Unità di Roma 1 and Center for Statistical Mechanics and Complexity, Roma 00185, Italy

${ }^{\ddagger}$ Los Alamos National Laboratory, Los Alamos, NM 87545

§Dipartimento di Fisica, Università di Roma "La Sapienza", Piazzale A. Moro 2, Roma 00185, Italy

ฯIstituto Nazionale per la Fisica della Materia, Unità di Roma 1 and Center for Statistical Mechanics and Complexity, Roma 00185, Italy

"Istituto Nazionale di Fisica Nucleare, Sezione di Roma 1, Roma 00185, Italy
} 


\section{Introduction}

Superfluidity and superconductivity are phenomena at the heart of quantum mechanics of many-body systems. Their importance is not limited to condensed matter physics and some of the concepts involved in their understanding have been seminal in other contexts, most notably in quantum field theory 11. A wealth of experimental informations on macroscopic quantum transport has been collected by studying the Bose and Fermi isotopes of helium in the superfluid phase and the electron liquids in superconductors [2. With the advent of Bose-Einstein condensates of dilute gases consequent to the development of innovative cooling technologies [3, 4, 5, 6, the class of systems available for studying superfluidity has been enlarged to many other species, namely various isotopes and hyperfine states of alkalis as well as atomic hydrogen, metastable helium, and ytterbium. Besides the richer spectrum of investigable atomic systems, in the case of dilute gases one can exploit their intrinsically slower dynamics - low densities imply weak average interactions - to study formation and decay of interesting structures like vortices, a possibility very hard to achieve in the denser liquid helium. Moreover, unlike liquid helium, the precision achievable with atomic physics experimental techniques, and the possibility to control the theoretical many-body approximations, are two other reasons which have contributed to the fast development of this sector at the borderline between atomic and condensed matter physics.

After the pioneering observation of Bose-Einstein condensation (BEC) in dilute gases of ${ }^{87} \mathrm{Rb}$ [7, ${ }^{23} \mathrm{Na}$ [8], and ${ }^{7} \mathrm{Li}$ [], a lot of experimental and theoretical activities have been focused on the signatures of superfluid behaviour in this novel low-density state of matter (see for instance 10 11, 12 ). Examples are the formation of vortices by means of optomechanical driving [13] and mechanical stirring [14] 15], the spectroscopy of scissor modes [16], the studies of superfluid flow and the related onset of a critical velocity [17, 18. These experimental achievements have been complemented by relatively simple, first-principle theoretical studies with significant progress in the comprehension of longstanding issues in liquid ${ }^{4} \mathrm{He}$, like critical velocities and vortex formation [19, 20. Even more interesting is the study, still completely open from the experimental viewpoint, of superfluid features in Fermi dilute gases [21. The presence of a superfluid phase is expected to occur in the deep degenerate regime via a sort of atomic Cooper pairing [22, 23], on the basis of qualitative analogies to the case of ${ }^{3} \mathrm{He}$ and, more in general, to high density electron liquids in superconductors.

In this paper we discuss in some length a recent proposal to confine and cool two-species Fermi and Bose gases in a bichromatic optical dipole trap 24]. This configuration allows for selective trapping of the two species with 
different trapping strengths. Since the confinement determines the degeneracy conditions, a regime can be chosen such that the Fermi temperature is much larger than the Bose-Einstein condensation temperature. In this case, a deep Fermi degeneracy could be achieved before (or in proximity) of the BEC phase-transition for the Bose component, leading to various advantages in the search for superfluidity in dilute fermions amidst a thermal (or thermally-dominated) Bose gas. This would provide an unprecedented situation as compared to the already available ${ }^{3} \mathrm{He}-{ }^{4} \mathrm{He}$ mixture. In principle, many Fermi-Bose mixtures can be studied. We will focus the discussion on the best two Bose coolers available, ${ }^{23} \mathrm{Na}$ and ${ }^{87} \mathrm{Rb}$ - the workhorses for Bose-Einstein condensation - and the only two stable Fermi isotopes for alkali atoms, ${ }^{6} \mathrm{Li}$ and ${ }^{40} \mathrm{~K}$.

The paper is organized as follows. In Section 2 we briefly describe the experimental techniques specific to the trapping and cooling of Fermi dilute gases, and then give an updated overview of the current experimental efforts in reaching Fermi degeneracy. In Section 3 we introduce our proposal by discussing the conservative trapping features in various configurations and for diverse combinations of Fermi-Bose mixtures. In Section 4 we discuss evaporative cooling for the boson species by giving a specific example of its dynamics in the case of one-color and two-color single-beam optical dipole traps. In Section 5 we deal with sympathetic cooling, with particular regard to the limitations to the minimum achievable temperatures induced by the heat capacities of the two species. In the same Section, we discuss qualitatively also some methods to evidence a possible superfluid state in the Fermi component, and comment on the advantages of having a thermal Bose cloud rather than the only Bose condensed component. General features of our proposal are summarized and discussed in the conclusions.

\section{Status of the experimental searches for su- perfluidity in dilute Fermi gases}

As a general consequence of the Heisenberg principle, quantum degeneracy occurs at temperatures very similar for bosons and fermions in presence (or absence) of external confining potentials with comparable strength. It is therefore natural to apply to the Fermi gases the successful trapping and cooling techniques already developed for bosons and culminating in the observation of a Bose condensed phase. However, there are also very striking differences between bosons and fermions in the degenerate regime. For instance, fermions enter into a degenerate regime without the sharp phase transition characteristic of bosons. Also, when dealing with Fermi systems one has always to face the effects of the Pauli principle which freezes most of the available degrees of freedom. The effects of the Pauli principle are 
particularly felt in all the current experimental efforts to achieve full degeneracy and to evidence a superfluid phase in Fermi systems confined by means of magnetic traps. With this trapping technique only spin-polarized Fermi gases can be confined and cooling is ultimately obtained - like in their bosonic counterparts - by the selective removal of the most energetic part of the atomic cloud allowing for rethermalization of the remaining fraction. Consequently, since the atoms are polarized in the same hyperfine state, the Pauli principle forbids the $s$-wave elastic scattering. Then rethermalization becomes inefficient at low temperature where the contribution of odd angular momentum states (like $p$-wave scattering) is strongly suppressed, and this limits the efficiency of direct evaporative cooling among fermions in the same hyperfine state. Two routes to overcome this limitation have been implemented, dual evaporative cooling of fermions prepared in two different hyperfine states, and sympathetic cooling with a Bose refrigerant. Even in these situations the Pauli principle gives limitations, as the elastic scattering between different hyperfine states is inhibited by the so-called Pauli blocking [25]: the atoms available to elastic scattering are limited to the Fermi surface and their number is directly proportional to $T / T_{\mathrm{F}}$. The use of sympathetic cooling with a boson reservoir is instead obstacled by the superfluidity of the latter [26], the fact that the specific heat of the Bose gas quickly vanishes fot $T<T_{\mathrm{c}}$, and ultimately by Pauli blocking. As a matter of fact, the lowest temperatures presently achieved for fermions are limited in the 0.05-0.2 $T_{\mathrm{F}}$ range [27, 28, 29, 30, 31, 32, 33, 34, 35, 36, 37.

Concerning the trapping techniques, the use of magnetic traps gives limitations in the combinatorics of trappable hyperfine states and interferes with the use of tunable homogeneous magnetic fields required to enhance atomic scattering via Feshbach resonances as predicted in 38. (see also 39. for a recent review) and observed in various atomic systems [40, 41, 42, 43]. Feshbach resonances provide a mechanism that could be crucial to identify signatures of superfluidity even at relatively large temperatures, the socalled resonant superfluidity [4, 45, 46, 47.

Some of the above mentioned limitations can be overcome by using optical dipole traps [48, 49, 50, 51]. Both different hyperfine states and arbitrary magnetic fields can be used in this case. Optical dipole traps have been pursued as a way to obtain quantum degeneracy with purely optical tools, avoiding the complications of magnetic trapping [52. After studies on degenerate Bose gases generated in magnetic traps and then transferred into optical dipole traps [53, both BEC [54, 55] and Fermi degeneracy [29] have been achieved directly in all-optical traps. Also, preliminary studies of Feshbach resonances for fermions in an optical trap have been reported in [56. More recently, studies of strongly interacting Fermi gases have been reported in the case of Fermi-Bose mixtures [57] and two-component Fermi gases [58, 59]. In the latter case the expansion dynamics has been 
interpreted as a possible evidence of resonant superfluidity as predicted in 60.

The experimental situation, updated to the Summer 2003, is summarized in Table 1. The two stable fermionic species ${ }^{6} \mathrm{Li}$ and ${ }^{40} \mathrm{~K}$ have been

\begin{tabular}{crll}
\hline \hline Atomic species & $N_{\mathrm{F}}$ & \multicolumn{1}{l}{$T / T_{\mathrm{F}}$} & Ref. \\
\hline${ }^{40} \mathrm{~K}(9 / 2,9 / 2)-{ }^{40} \mathrm{~K}(9 / 2,7 / 2)$ & $7 \times 10^{5}$ & 0.50 & JILA1 [25] \\
${ }^{6} \mathrm{Li}(3 / 2,3 / 2)-{ }^{7} \mathrm{Li}(2,2)$ & $1.4 \times 10^{5}$ & 0.25 & Rice1 27] \\
${ }^{6} \mathrm{Li}(3 / 2,3 / 2)-{ }^{7} \mathrm{Li}(2,2)$ & $4 \times 10^{3}$ & 0.20 & ENS1 [28] \\
${ }^{6} \mathrm{Li}(1 / 2,1 / 2)-{ }^{6} \mathrm{Li}(1 / 2,-1 / 2)$ & $10^{5}$ & 0.50 & Duke1 [29] \\
${ }^{6} \mathrm{Li}(1 / 2,1 / 2)-{ }^{23} \mathrm{Na}(1,-1)$ & $1.4 \times 10^{5}$ & 0.50 & MIT1[30] \\
${ }^{40} \mathrm{~K}(9 / 2,9 / 2)-{ }^{87} \mathrm{Rb}(2,2)$ & $10^{4}$ & 0.30 & LENS 31] \\
${ }^{6} \mathrm{Li}(1 / 2,1 / 2)-{ }^{6} \mathrm{Li}(1 / 2,-1 / 2)$ & $1.6 \times 10^{5}$ & 0.15 & Duke2 [32] \\
${ }^{40} \mathrm{~K}(9 / 2,-9 / 2)-{ }^{40} \mathrm{~K}(9 / 2,-5 / 2)$ & $1.1 \times 10^{6}$ & 0.21 & JILA2 [33] \\
${ }^{6} \mathrm{Li}(3 / 2,3 / 2)-{ }^{7} \mathrm{Li}(2,2)$ & $7 \times 10^{7}$ & 0.10 & Rice2 [34] \\
${ }^{6} \mathrm{Li}(1 / 2,1 / 2)-{ }^{23} \mathrm{Na}(2,2)$ & $7 \times 10^{7}$ & 0.05 & MIT2 [35] \\
${ }^{6} \mathrm{Li}(1 / 2,-1 / 2)-{ }^{6} \mathrm{Li}(1 / 2,1 / 2)$ & $8 \times 10^{4}$ & 0.43 & ENS2 [36] \\
\hline \hline
\end{tabular}

Table 1: Status of the experimental studies of degenerate Fermi dilute gases. For each laboratory the various trapped species are reported specifying the particular hyperfine state $\left(F, m_{F}\right)$, the number of fermions at the final stage of cooling $N_{\mathrm{F}}$, the lowest reached temperature ratio $T / T_{\mathrm{F}}$, and the related reference. The quoted experiments at JILA and Duke University make use of dual evaporative cooling of two hyperfine states of magnetically trapped potassium and optically trapped lithium, respectively. The other experiments exploit sympathetic cooling with bosonic reservoirs, ${ }^{7} \mathrm{Li},{ }^{23} \mathrm{Na}$, and ${ }^{87} \mathrm{Rb}$. More recently, sympathetic cooling of ${ }^{40} \mathrm{~K}$ with ${ }^{87} \mathrm{Rb}$ has been also pursued at JILA [37. In subsequent work the Duke University group has reached deeper degeneracy and observed an anisotropic expansion of the Fermi gas, which could be interpreted as an evidence of superfluid behavior of the Fermi gas [58, 59]. More recent experiments use Feshbach resonances, resulting in high-efficiency production of potassium or lithium ultracold molecules. In the experiment described in [34 the Fermi-Bose mixture is an intermediate stage, then all the bosons are removed and the fermions are prepared in an incoherent mixture of equal populations in the $(1 / 2,-1 / 2)$ and $(1 / 2,1 / 2)$ states.

cooled by using evaporative cooling between two hyperfine states (JILA and Duke University) or through sympathetic cooling with Bose condensates of ${ }^{7} \mathrm{Li},{ }^{23} \mathrm{Na}$, and ${ }^{87} \mathrm{Rb}$ (Rice-ENS, MIT, and LENS respectively). It is evident that, despite of very different trapping and cooling techniques, the lowest degeneracy parameter $T / T_{\mathrm{F}}$ obtained by using sympathetic cooling 
is around 0.2 (for the particular case of MIT2 see a detailed discussion in Section 5). The existence of this sort of lower bound for $T / T_{\mathrm{F}}$ can be understood semiquantitatively in the following way [61. In the latest stage of evaporative cooling the number of Bose atoms becomes of the same order

of magnitude of the number of Fermi atoms (in some hyperfine states of ${ }^{7} \mathrm{Li}$, due to the negative scattering length, there is also a theoretical upper limit to the number of atoms in the condensed phase, see [9]). On the other hand the boson specific heat scales as $T^{3}$ below the BEC transition, while the Fermi atoms have specific heat scaling as $T$. The specific heat curves of bosons and fermions, assuming for simplicity equal masses for the two species, intersect each other at a temperature $T^{*} \sim 0.5 T_{\mathrm{c}} \sim 0.25 T_{\mathrm{F}}$. Below $T^{*}$ sympathetic cooling becomes very inefficient. This simple explanation gives also an hint on how to overcome the limitation. If one could engineer the trapping potential in such a way that $T_{\mathrm{c}} \ll T_{\mathrm{F}}$, the Bose gas would preserve enough thermal capacity to drive the sympathetic cooling even in a deep degenerate regime for the Fermi component. A first attempt in this direction can be found in [62, where adiabatic compression was proposed in an optical dipole trap superimposed to an already confining magnetic trap. This should allow a small fraction of the Fermi atoms to experience a very tight confinement potential, thus enhancing the Fermi temperature. More recently, we have proposed an alternative solution in the context of pure optical trapping [24], by using a two-color optical dipole trap which confines both Fermi and Bose gases with different strengths. It is the purpose of the next Section to discuss in detail the static confinement features of this class of atomic traps.

\section{Two-color optical dipole traps}

Let us start our analysis from the conditions of degeneracy for Fermi and Bose gases confined in harmonic traps. The Fermi and Bose temperatures for dilute atomic clouds trapped by harmonic potentials can be written as:

$$
\begin{gathered}
T_{\mathrm{F}}=6^{1 / 3} \hbar \omega_{\mathrm{f}} N_{\mathrm{f}}^{1 / 3} k_{\mathrm{B}}^{-1} \simeq 1.82 \hbar \omega_{\mathrm{f}} N_{\mathrm{f}}^{1 / 3} k_{\mathrm{B}}^{-1} \\
T_{\mathrm{c}}=\zeta(3)^{-1 / 3} \hbar \omega_{\mathrm{b}} N_{\mathrm{b}}^{1 / 3} k_{\mathrm{B}}^{-1} \simeq 0.94 \hbar \omega_{\mathrm{b}} N_{\mathrm{b}}^{1 / 3} k_{\mathrm{B}}^{-1}
\end{gathered}
$$

with $\omega_{\mathrm{f}}=\left(\omega_{\mathrm{f} x} \omega_{\mathrm{f} y} \omega_{\mathrm{f} z}\right)^{1 / 3}$ and $\omega_{\mathrm{b}}=\left(\omega_{\mathrm{b} x} \omega_{\mathrm{b} y} \omega_{\mathrm{b} z}\right)^{1 / 3}$ being the geometrical average of the angular trapping frequencies in the three directions for fermions and bosons, $N_{\mathrm{f}}$ and $N_{\mathrm{b}}$ the number of atoms of the Fermi and Bose gases, and $\hbar$ and $k_{\mathrm{B}}$ the Planck and Boltzmann constants, respectively.

Besides the small difference in the prefactor, the degenerate temperatures for Fermi and Bose atoms per unit of atom are similar in traps with 
size of eps file too large, not accepted by arXiv

Curious to see the figure? look at the published paper

http://dx.doi.org/10.1023/B:JOSS.0000019829.71660.40

Figure 1: Combined optical dipole trap for two-species mixtures. Case of a single beam configuration (left) with the two color beams propagating in a coaxial fashion, and of a coplanar crossed-beam dipole trap (right) with relative angle between the two beam pairs $\theta=0$. The lenses are assumed to be achromatic to ensure a common focus for the collimated beams at different wavelengths.

the same angular trapping frequencies for the two species. This is indeed the case of magnetic traps: since the magnetic moments of the alkalimetals are very similar, the only difference in the trapping strengths is due to their different masses, with the angular trapping frequencies scaling as $\omega_{\mathrm{f}} / \omega_{\mathrm{b}} \simeq\left(m_{\mathrm{b}} / m_{\mathrm{f}}\right)^{1 / 2}$. The situation may change in optical dipole traps where the confinement is dictated by the detunings of the laser beams with respect to the atomic transitions and by the beam intensities. Situations for which Fermi degeneracy is reached before BEC (i.e. $T_{\mathrm{F}}>T_{\mathrm{c}}$ ) are therefore viable, provided that fermions and bosons have different atomic transitions. This will restrict our analysis to two-species mixtures, since the isotopic shifts are usually not enough to ensure selective trapping in single species Fermi-Bose mixtures without incurring in prohibitive heating due to residual Rayleigh scattering.

In discussing optical dipole traps, one can consider either single beam or crossed-beam configurations [52, see Figure 1. The former has the advantage of being simpler with fewer experimental problems of loading and alignment, the latter gives rise to a more isotropic confinement. In the following, we will discuss in detail the crossed-beam geometry. Results for the single beam configuration will be obtained as a particular case in which one of the two beams is turned off.

In the crossed-beam configuration, a pair of laser beams red-detuned 
with respect to the atomic transitions and focused on the center of the preexisting trapping potential (for instance the one generated by the magnetooptical trap typically used for precooling the atomic clouds), gives an effective attractive potential for both the species. This attractive potential is partially balanced by a second pair of mutually orthogonal blue-detuned laser beams, acting for instance along the same plane formed by the reddetuned beams and forming with the latter an angle $\theta$. This second pair of beams gives rise to a repulsive potential and, by a proper choice of its detuning and power, provides a selective deconfinement for the two species.

The potential generated by the dynamical Stark effect, felt by an atom of species $\alpha$ ( $\alpha=\mathrm{b}$ for bosons, $\alpha=\mathrm{f}$ for fermions) whose atomic transition wavelength and linewidth are respectively $\lambda_{\alpha}$ and $\Gamma_{\alpha}$, and due to the laser beams $i$ of wavelength $\lambda_{i}$ and intensity $I_{i}(i=1,2$ for the red-detuned and blue-detuned laser beams, respectively), is 48

$$
U_{i}^{\alpha}(x, y, z)=-\frac{\hbar \Gamma_{\alpha}^{2}}{8 I_{\alpha}^{\text {sat }}}\left(\frac{1}{\Omega_{\alpha}-\Omega_{i}}+\frac{1}{\Omega_{\alpha}+\Omega_{i}}\right) I_{i}(x, y, z),
$$

where $\Omega_{\alpha}=2 \pi c / \lambda_{\alpha}, \Omega_{i}=2 \pi c / \lambda_{i}$, and $I_{\alpha}^{\text {sat }}$ is the saturation intensity for the atomic transition, expressed in terms of the former quantities as $I_{\alpha}^{\text {sat }}=\hbar \Omega_{\alpha}^{3} \Gamma_{\alpha} / 12 \pi c^{2}$. It is easy to recognize that the potential energy has the same sign of the laser intensity if $\Omega_{i}>\Omega_{\alpha}$, i.e. for blue-detuned light. Then the atoms, trying to minimize their potential energy, move towards the regions of space with minimum light intensity, and therefore are expelled by the laser beam. The opposite occurs for red-detuned light, for which the atoms are attracted in the regions of maximum light intensity.

The laser intensity is the incoherent sum of the intensities of each pair of beams (such incoherent sum can be obtained by orthogonal polarizations or by slight detuning of the beams within each pair), all focused at the origin of the trap. If we assume that the red-detuned beams propagate along the $x-y$ axes while the blue-detuned beams propagate along the $\xi-\eta$ axes possibly rotated by an angle $\theta$, i.e. $\xi=x \cos \theta+y \sin \theta, \eta=y \cos \theta-x \sin \theta$, with $0 \leq \theta<\pi / 4$, the intensities can be written as

$$
\begin{aligned}
& I_{1}(x, y, z)=\frac{2 P_{1}}{\pi w_{1}^{2}}\left\{\frac{\exp \left[-\frac{2\left(y^{2}+z^{2}\right)}{w_{1}^{2}\left(1+x^{2} / R_{1}^{2}\right)}\right]}{1+x^{2} / R_{1}^{2}}+\frac{\exp \left[-\frac{2\left(x^{2}+z^{2}\right)}{w_{1}^{2}\left(1+y^{2} / R_{1}^{2}\right)}\right]}{1+y^{2} / R_{1}^{2}}\right\} \\
& I_{2}(x, y, z)=\frac{2 P_{2}}{\pi w_{2}^{2}}\left\{\frac{\exp \left[-\frac{2\left(\eta^{2}+z^{2}\right)}{w_{2}^{2}\left(1+\xi^{2} / R_{2}^{2}\right)}\right]}{1+\xi^{2} / R_{2}^{2}}+\frac{\exp \left[-\frac{2\left(\xi^{2}+z^{2}\right)}{w_{2}^{2}\left(1+\eta^{2} / R_{2}^{2}\right)}\right]}{1+\eta^{2} / R_{2}^{2}}\right\}
\end{aligned}
$$

where $P_{i}$ is the laser power, $w_{i}$ is the $1 / e^{2}$ beam waist radius, and $R_{i}=$ $\pi w_{i}^{2} / \lambda_{i}$ the Rayleigh range. The quantity $2 P_{i} / \pi w_{i}^{2}=I_{i}(0,0,0)$ represents 
the peak laser intensity due to each pair of beams, obtained in the focal point.

The overall potential felt by the fermions (bosons) is $U_{\mathrm{f}}=U_{1}^{\mathrm{f}}+U_{2}^{\mathrm{f}}$ $\left(U_{\mathrm{b}}=U_{1}^{\mathrm{b}}+U_{2}^{\mathrm{b}}\right)$. For a proper choice of the laser powers, these potentials are energy wells with depths $\Delta U_{\mathrm{f}}$ and $\Delta U_{\mathrm{b}}$ which constitute the confining energies of the fermionic and bosonic species. The curvatures around the minimum of these potentials determine the trapping frequencies $\omega_{\mathrm{f}}$ and $\omega_{\mathrm{b}}$ and, consequently, the critical temperatures $T_{\mathrm{F}}$ and $T_{\mathrm{c}}$. Up to quadratic terms, the potentials $U_{\mathrm{f}}$ and $U_{\mathrm{b}}$ are invariant under rotations around the minimum so that the trapping frequencies do not depend on the angle $\theta$. By neglecting the terms $\left(\lambda_{i} / \pi w_{i}\right)^{2}<<1$, we find

$$
\omega_{\alpha x}=\omega_{\alpha y}=\frac{\omega_{\alpha z}}{\sqrt{2}}=\sqrt{\frac{\hbar}{\pi m_{\alpha}}\left(\frac{k_{1}^{\alpha} P_{1}}{w_{1}^{4}}+\frac{k_{2}^{\alpha} P_{2}}{w_{2}^{4}}\right)},
$$

where the constants $k_{i}^{\alpha}$ have been introduced as

$$
k_{i}^{\alpha}=\frac{\Gamma_{\alpha}^{2}}{I_{\alpha}^{\text {sat }}}\left(\frac{1}{\Omega_{\alpha}-\Omega_{i}}+\frac{1}{\Omega_{\alpha}+\Omega_{i}}\right) .
$$

Analogous expressions can be obtained in the case of a single-beam geometry. For instance, by considering a single beam propagating along the $x$-axis we have

$$
\begin{gathered}
\omega_{\alpha x}=\sqrt{\frac{\hbar}{\pi m_{\alpha}}\left(\frac{k_{1}^{\alpha} P_{1}}{w_{1}^{2} R_{1}^{2}}+\frac{k_{2}^{\alpha} P_{2}}{w_{2}^{2} R_{2}^{2}}\right)}, \\
\omega_{\alpha z}=\omega_{\alpha y}=\sqrt{\frac{\hbar}{\pi m_{\alpha}}\left(\frac{k_{1}^{\alpha} P_{1}}{w_{1}^{4}}+\frac{k_{2}^{\alpha} P_{2}}{w_{2}^{4}}\right) .}
\end{gathered}
$$

Since typically $w_{i} \gg \lambda_{i}$ and, therefore, $R_{i} \gg w_{i}$, the confinement along the $x$-direction is weakened leading, with respect to the crossed-beam configuration, to an overall reduction of the average angular frequencies for the trapped species. In the particular case of equal ratios between wavelengths and waists for the two colors, $\lambda_{1} / w_{1}=\lambda_{2} / w_{2}=\lambda / w$, this reduction amounts to $(\lambda / 2 \pi w)^{1 / 3}$.

In Figure 2 we show the dependence of the optical potential upon the radial, $x$ or $y$, and axial, $z$, directions in the two-beam geometry for the case of the ${ }^{6} \mathrm{Li}^{23} \mathrm{Na}$ mixture. When the ratio between the power of the blueand red-detuned lasers is small $\left(P_{2} / P_{1}=0.05\right.$, upper panels) the deformations induced by the blue-detuned beam are negligible. The difference in curvature between the two species is mainly attributable to the difference in mass $m_{\alpha}$ and detuning with respect to the laser beam, both playing a role in favoring $T_{\mathrm{c}}$ smaller than $T_{\mathrm{F}}$. Also, the depth of the potential well for 

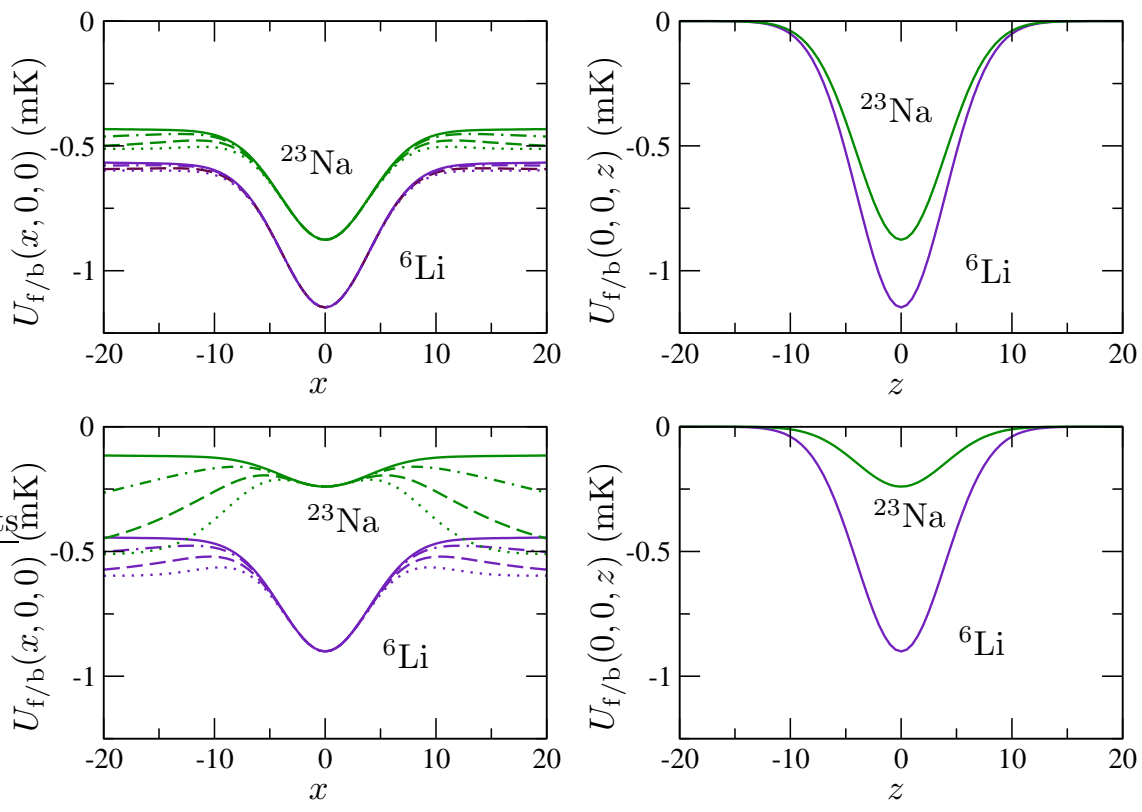

Figure 2: Potential energies in the case of the ${ }^{6} \mathrm{Li}^{23} \mathrm{Na}$ mixture in a bichromatic optical dipole trap in a crossed-beam geometry formed by focusing a Nd:YAG laser beam $\left(\lambda_{1}=1064 \mathrm{~nm}\right)$ and a blue-detuned laser beam at its second harmonic $\left(\lambda_{2}=532 \mathrm{~nm}\right)$. In the two upper panels the potential energy for the atoms of the two species is shown along the radial, $x$ or $y$, and axial, $z$, directions for a power ratio $P_{2} / P_{1}=0.05$. The four curves in the radial directions refer to various angles $(\theta=0, \pi / 16, \pi / 8$, and $\pi / 4$ from top to bottom) between the two pairs of beams. In the two lower panels the case of a larger power ratio, $P_{2} / P_{1}=0.25$, is considered. The beam waists are $w_{1}=w_{2}=8 \mu \mathrm{m}$, and $P_{1}=1 \mathrm{~W}$. Note that while the potential vanishes with a purely Gaussian behaviour in the axial direction $z$, along $x$ (and $y$ ) first it varies in a Gaussian way (to half of its peak value for $\theta=0$ ) and finally vanishes as a Lorentzian (not shown). The Gaussian and Lorentzian widths are determined by the beam waists and the Rayleigh ranges, respectively. 


\begin{tabular}{cclclc}
\hline \hline mixture & $\lambda_{\mathrm{f}}(\mathrm{nm})$ & $\Gamma_{\mathrm{f}}(\mathrm{MHz})$ & $\lambda_{\mathrm{b}}(\mathrm{nm})$ & $\Gamma_{\mathrm{b}}(\mathrm{MHz})$ & $\lambda_{2}(\mathrm{~nm})$ \\
\hline${ }^{6} \mathrm{Li}^{23} \mathrm{Na}$ & 671 & $2 \pi \times 5.9$ & 589 & $2 \pi \times 9.8$ & 532 \\
${ }^{6} \mathrm{Li}^{87} \mathrm{Rb}$ & 671 & $2 \pi \times 5.9$ & 780 & $2 \pi \times 5.98$ & 740 \\
${ }^{40} \mathrm{~K}^{23} \mathrm{Na}$ & 767 & $2 \pi \times 6.09$ & 589 & $2 \pi \times 9.8$ & 532 \\
${ }^{40} \mathrm{~K}^{87} \mathrm{Rb}$ & 767 & $2 \pi \times 6.09$ & 780 & $2 \pi \times 5.98$ & 773.5 \\
\hline \hline
\end{tabular}

Table 2: Wavelenghts and linewidths of the atomic transitions for the fermion-boson mixtures considered in the text and wavelength of the corresponding deconfining laser with a Nd:YAG laser at $\lambda_{1}=1064 \mathrm{~nm}$ producing the primary trapping potential.

the bosonic species is smaller than that for the fermions. This makes possible to exploit evaporative cooling without appreciable interference from the Fermi cloud. It is also evident that the stronger confinement in the radial direction is achieved for coaxial beams, i.e. $\theta=0$. The axial confinement along the $z$ axis is instead unaffected by the rotation angle between the beam pairs. In the case of a strong perturbation, lower panels of Fig. 2 where $P_{2} / P_{1}=0.25$, the bosonic species is much less confined and there is a strong difference in the curvature of the energy potential with respect to the fermionic species.

In both crossed- or single-beam configurations, the confinement energy of the boson species vanishes as the power ratio $P_{2} / P_{1}$ approaches a critical value. Approximately, this can be obtained by Eq. (6) or Eqs. (89) as the $P_{2} / P_{1}$ value at which the average trapping frequency of bosons vanishes. In both cases, we find

$$
\left.\frac{P_{2}}{P_{1}}\right|_{\text {crit }}=\frac{\Omega_{2}^{2}-\Omega_{\mathrm{b}}^{2}}{\Omega_{\mathrm{b}}^{2}-\Omega_{1}^{2}}\left(\frac{w_{2}}{w_{1}}\right)^{4} .
$$

The behavior of the trapping frequency ratio $\omega_{\mathrm{f}} / \omega_{\mathrm{b}}$ as well as of the trapping energies $\Delta U_{\mathrm{f}}$ and $\Delta U_{\mathrm{b}}$ as a function of the power ratio $P_{2} / P_{1}$ is shown in Figs. 3 and 4 also for different Fermi-Bose mixtures, obtained by using fermionic ${ }^{40} \mathrm{~K}$ and bosonic ${ }^{87} \mathrm{Rb}$, as summarized in Table 2 . The trapping frequency ratio for the single-beam geometry is always reduced with respect to the corresponding crossed-beam case at the same power ratio $P_{2} / P_{1}$. This decrease, which is a consequence of the weaker confinement along the direction of the laser beam, can be compensated by a larger power ratio, provided that the beam intensities are adequately stabilized. From the upper panels in Figure 4 we see that, with the chosen values of the blue-detuned laser wavelength, the bosonic sodium atoms are always less confined than the fermionic atoms. This allows one to apply evaporative cooling techniques for the bosonic species while having negligible losses 

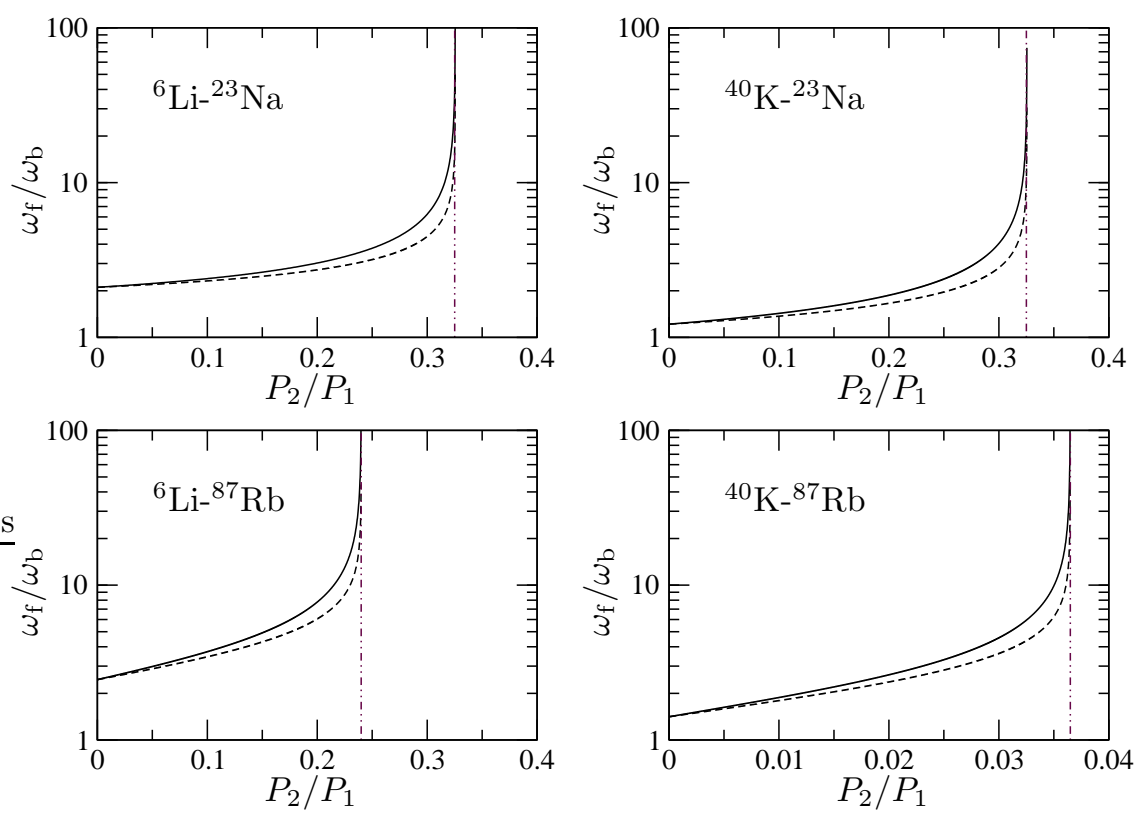

Figure 3: Selective trapping of bosons and fermions. The ratio between the average trapping frequencies for the fermionic and bosonic species is shown versus the beam power ratio between the blue- and red-detuned lasers. The cases of single beam (dashed lines) and crossed beam (solid lines) geometries are depicted for the $\mathrm{Li}-\mathrm{Na}$, Li-Rb, K-Na, and $\mathrm{K}-\mathrm{Rb}$ mixtures. The vertical lines are the critical values $P_{2} / P_{1}$ given by Eq. (10). Parameters chosen according to the values in Table 2 . 

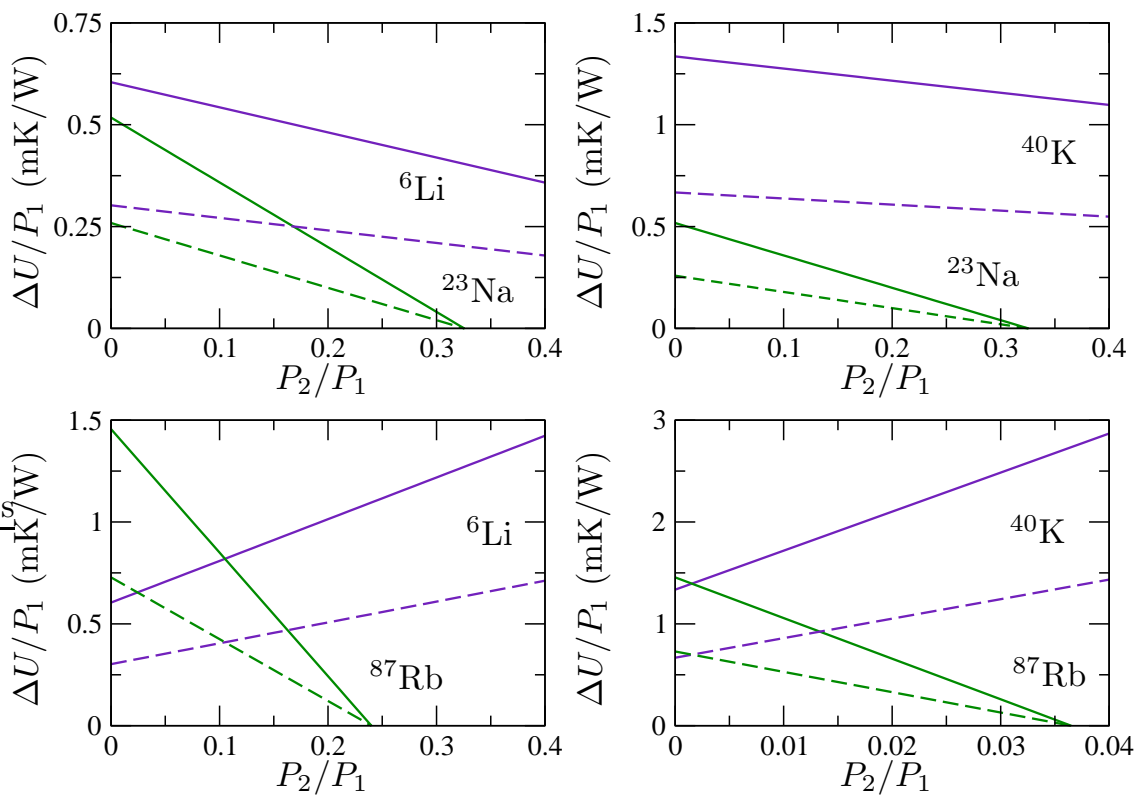

Figure 4: Selective trapping of bosons and fermions. Confining energy per unit of infrared laser power as a function of the beam power ratio between the blue- and red-detuned lasers. The confining energy is evaluated with respect to the region of space delimited by the waist size (see also note in the caption of Figure 2). Continuous lines refer to the crossed-beam configuration, while dashed lines are for the single-beam optical dipole trap. 
in the number of fermions. For the mixtures utilizing ${ }^{87} \mathrm{Rb}$, the confining energy is initially higher for the Bose species (especially for the Li-Rb mixtures), however the fermionic confining energy increases as the laser power $P_{2}$ is increased, since the potential created by the blue-detuned light is in this case attractive for fermions $\left(\lambda_{2}>\lambda_{\mathrm{f}}\right)$. For these mixtures, therefore, the addition of the laser light deconfining the bosons improves at the same time the confinement features of the fermionic species and minimizes the losses of Fermi atoms at the beginning of evaporation. Note also that for all the mixtures considered, within a very good approximation the bosonic confinement energies $\Delta U_{\mathrm{b}}$ in Figure 4 vanish at the same critical values $P_{2} / P_{1}$ at which the ratios $\omega_{\mathrm{f}} / \omega_{\mathrm{b}}$ in Figure 3 diverge.

Some further comments are in order. In the case of equal waists, the potential felt by the bosons is exactly zero at the critical power ratio and changes curvature at larger values giving rise to deconfinement, see middle panel of Figure 5. By choosing $w_{2}<w_{1}$ (top panel of Figure 5), the potential becomes bistable for $P_{2} / P_{1}$ around the critical value. Then, the blue-detuned laser can be used to produce tailored bistable potentials, providing an alternative route to the recently demonstrated all-magnetic bistable potentials 63]. For $w_{2}>w_{1}$ (bottom panel of Figure 5), the potential is always globally deconfining but a local minimum around the origin is assured if $P_{2} / P_{1}$ is close to the critical value. The strong dependence of the critical power ratio upon the beam waists can be used to reduce the amount of blue-detuned light necessary to approach the targeted values of $\omega_{\mathrm{f}} / \omega_{\mathrm{b}}$. However, based on the above mentioned considerations, there is a tradeoff since we may have also a change of the potential shape.

A possible technical issue is the demand for an initial large laser power especially since it is difficult to get sub-Doppler cooling for Li and $\mathrm{K}$ due to the small hyperfine splittings of the $p_{3 / 2}$ atomic level 64 (see however [65] for a successful demonstration of sub-Doppler cooling of ${ }^{40} \mathrm{~K}$ ). Up to now, Nd:YAG laser powers with built-in crystals for frequency-doubling are limited to about $1 \mathrm{~W}$. This corresponds to order of $500 \mu \mathrm{K}$ for the initial potential depth, i.e. four times the initial temperature of the cloud if the latter is transferred from a Doppler-limited magneto-optical trap. As we will discuss in the next Section, this could be an issue for starting efficient evaporative cooling. Besides awaiting progress in the power deliverable by semiconductor-based lasers, one can use independent systems for red- and blue-detuned light (this seems anyway unavoidable for mixtures utilizing ${ }^{87} \mathrm{Rb}$ as the Bose cooler). High power, far detuned $\mathrm{CO}_{2}$ lasers are also available to produce quasi-static optical dipole traps [66, 67. This technique has been recently demonstrated for cooling fermionic lithium at degeneracy temperature 29] after efficient loading from a magneto-optical trap 68 69. $\mathrm{CO}_{2}$ lasers allow for large powers (order of 100-200 W) and, since they are well detuned from the atomic resonances, for very small heating 

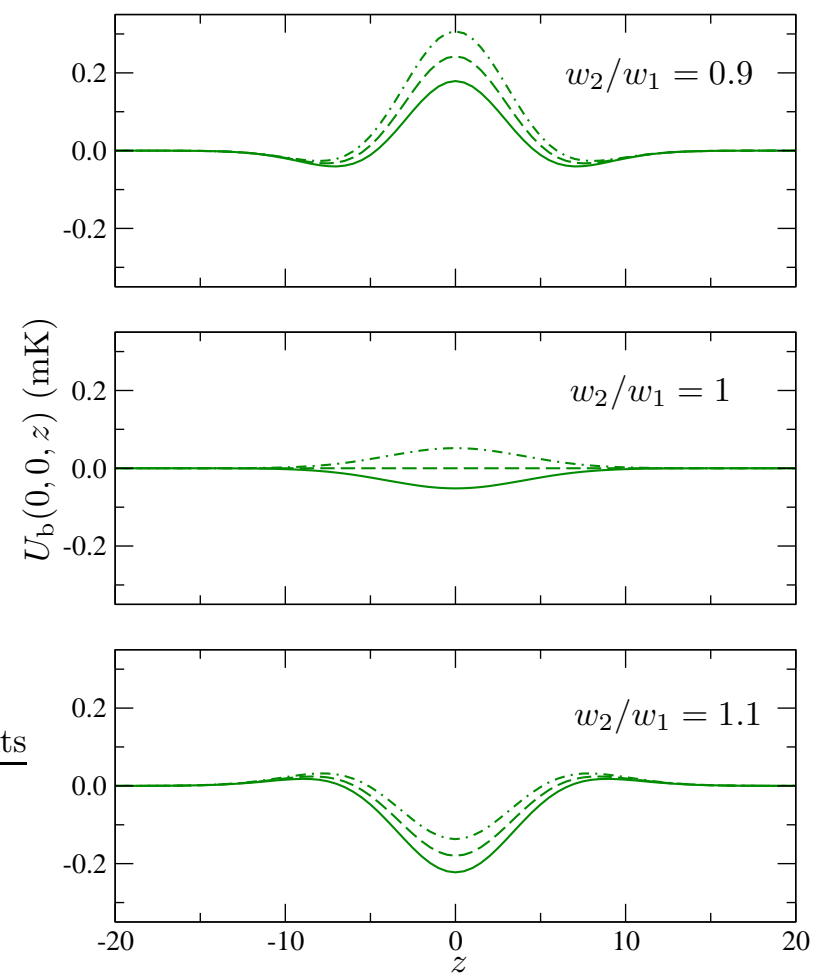

Figure 5: Bosonic potential energy along the $z$-axis in the case of the ${ }^{6} \mathrm{Li}^{23} \mathrm{Na}$ mixture in a crossed-beam bichromatic optical dipole trap for $P_{2} / P_{1} 5 \%$ smaller (solid), equal (dashed), and $5 \%$ larger (dot-dashed) than $P_{2} /\left.P_{1}\right|_{\text {crit }}$. The three panels refer to waist ratios $w_{2} / w_{1}=0.9$ (top), $w_{2} / w_{1}=1$ (middle), and $w_{2} / w_{1}=1.1$ (bottom). 

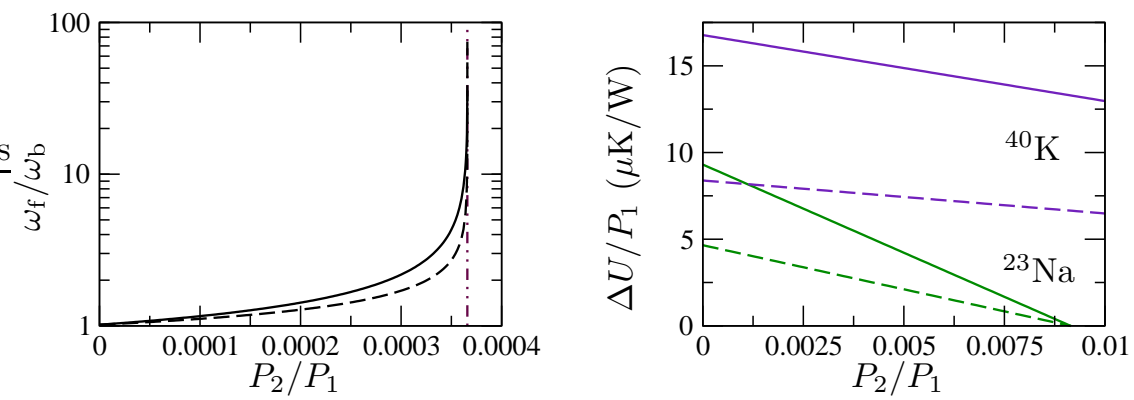

Figure 6: Selective trapping with a $\mathrm{CO}_{2}$ laser in the case of the $\mathrm{K}-\mathrm{Na}$ mixture. Trapping frequency ratio (left) and confinement energy (right) versus the parameter $P_{2} / P_{1}$. The dashed curves refer to the single-beam configuration, the continuous ones to the crossed-beam geometry. The different critical values of $P_{2} / P_{1}$ obtained from the left and right panels are due to the fact that the harmonic approximation used to evaluate $\omega_{\mathrm{f}} / \omega_{\mathrm{b}}$ fails for $P_{2} / P_{1}$ large enough (the potential becomes bistable since $w_{2}<w_{1}$ ). The waist of the $\mathrm{CO}_{2}$ laser beam is assumed to be $w_{1}=50 \mu \mathrm{m}$, while $w_{2}=10 \mu \mathrm{m}$.

due to photon scattering 70 . In Figure 6 we show the selective trapping features of a $\mathrm{K}-\mathrm{Na}$ mixture with the $\mathrm{Nd}$ :YAG laser replaced by a $\mathrm{CO}_{2}$ laser emitting at $10.6 \mu \mathrm{m}$. At such large wavelengths the optical potential is well approximated by the induced dipole interaction $U=-\alpha_{\mathrm{g}} \overline{E^{2}} / 2$, where $\alpha_{\mathrm{g}}$ is the ground state polarizability and $\overline{E^{2}}$ is the time-averaged squared electric field [66]. In terms of the laser intensity the optical potential is

$$
U(x, y, z)=-\frac{2 \pi \alpha_{\mathrm{g}}}{c} I(x, y, z)
$$

Due to the high static atomic polarizability of rubidium $\left(\alpha_{\mathrm{g}}^{\mathrm{Rb}}=47.3 \times\right.$ $10^{-24} \mathrm{~cm}^{3}$ ), this bosonic species is more strongly confined than fermionic potassium $\left(\alpha_{\mathrm{g}}^{\mathrm{K}}=43.4 \times 10^{-24} \mathrm{~cm}^{3}\right)$ and lithium $\left(\alpha_{\mathrm{g}}^{\mathrm{Li}}=24.3 \times 10^{-24} \mathrm{~cm}^{3}\right)$, therefore ruling out evaporation as a possible cooling technique for $\mathrm{K}-\mathrm{Rb}$ and $\mathrm{Li}-\mathrm{Rb}$ mixtures. Also, the combination Li-Na has to be discarded due to the small static atomic polarizability of sodium $\left(\alpha_{\mathrm{g}}^{\mathrm{Na}}=24.08 \times 10^{-24}\right.$ $\left.\mathrm{cm}^{3}\right)$. Thus, a $\mathrm{CO}_{2}$ laser can be effectively used for a red-detuned attractive potential only in the case of the ${ }^{40} \mathrm{~K}_{-}{ }^{23} \mathrm{Na}$ mixture considered in Figure 6. 


\section{Evaporative cooling}

So far we have considered the static trapping features of the two-color optical dipole configuration schematized through a time-independent potential. However, to reach quantum degeneracy, the phase space density has to be increased, e.g. by cooling down the atomic sample. In the configuration proposed here this is obtained through two continuous processes: forced and selective removal of bosonic atoms with re-thermalization of the surviving component (evaporative cooling) [6], and thermalization of the fermionic species to the temperature of the Bose gas (sympathetic cooling). These cooling processes have various limitations, the most obvious one being the reduced heat capacity of the bosonic sample undergoing a continuous decrease of atoms. Moreover, concurrent heating sources will limit the ultimate reachable temperature, most notably the residual Rayleigh scattering from the trapping beams. In this Section we discuss to some extent these issues and, although not pretending to go into full details on the many possible experimental schemes, we give estimates of the relevant parameters involved in the cooling dynamics for a specific example.

Evaporative cooling in an optical dipole trap has been the subject of various experimental and theoretical studies [52, 54, 71]. The atomic evaporation rate in finite-depth traps is exponentially dependent upon the ratio between the potential depth of the trap and the average thermal energy of the atomic cloud, $\Delta U / k_{\mathrm{B}} T$, since the number of atoms in the tail of the Boltzmann distribution scales as $\exp \left(-\Delta U / k_{\mathrm{B}} T\right)$. Forced evaporation is, therefore, necessary to maintain a significant evaporation rate, which should be otherwise exponentially quenched by reducing the cloud temperature. It can be demonstrated [71] that all the relevant quantities involved in forced evaporative cooling in an optical dipole trap scale with some power of the confining energy, provided that the ratio between the latter and the cloud temperature is constant in time $\Delta U / k_{\mathrm{B}} T=\eta$. In this case, the confining energy has a time dependence of the form

$$
\frac{\Delta U(t)}{\Delta U_{\mathrm{i}}}=\left(1+\frac{t}{\tau}\right)^{\varepsilon_{U}}
$$

where $\Delta U_{\mathrm{i}}$ is the initial potential depth, $\varepsilon_{U}=-2\left(\eta^{\prime}-3\right) / \eta^{\prime}$, and $\tau^{-1}=$ $(2 / 3) \eta^{\prime}(\eta-4) \exp (-\eta) \gamma_{\mathrm{i}}$, with $\eta^{\prime}=\eta+(\eta-5) /(\eta-4)$ and $\gamma_{\mathrm{i}}$ being the initial value of the elastic collision rate $\gamma$. Similar laws hold for the time dependence of the number of particles $N$, temperature $T$, phase-space density $\rho$, and elastic collision rate $\gamma$ with corresponding exponents $\varepsilon_{N}, \varepsilon_{T}, \varepsilon_{\rho}$, and $\varepsilon_{\gamma}$.

With respect to the simplest case of a single Bose species undergoing evaporative cooling in an optical dipole trap we have two differences. First, there is a mixture also containing the Fermi atoms. Their presence does 
not significantly affect evaporative cooling of the Bose gas if the potential energy depth $\Delta U_{\mathrm{f}}$ is much larger than $\Delta U_{\mathrm{b}}$, a condition well satisfied in the situations we consider - see Figure 4. Also, in order for the Bose gas to act as a cooler its heat capacity must be much larger than the heat capacity of the fermionic species. This is possible, at least in the earlier stages of evaporation before entering the degenerate regime, by assuming a number of bosons much larger than the number of fermions. In bichromatic traps, a further problem may arise due to the presence of the blue-detuned laser which weaken the confinement of both species, particularly the bosonic one. As the atomic densities are decreased, all the density-dependent scattering rates, in particular the elastic one which is crucial for thermalization of the surviving atoms, are also suppressed 72 .

The lower densities and the slower thermalization at lower temperatures makes more difficult to achieve high phase-space densities, an issue which has limited for various years the effort to make an all-optical formation of Bose-Einstein condensates 73 . From this point of view, in a bichromatic trap it is convenient to turn on the blue-detuned beam only when it can give an effective advantage, i.e. close to the Fermi degenerate regime. Once on, the power of the blue-detuned beam must be chosen in such a way to maintain a fixed ratio with respect to that of the red-detuned beam. Meanwhile the power of the red-detuned beam is decreased continuously to allow efficient forced evaporation of the bosonic species. An example of this cooling strategy is shown in Figure 7.

The constant power ratio $P_{2} / P_{1}$ must be chosen carefully as a compromise between increasing the fermion-to-boson trapping frequency ratio and not decreasing too much the absolute frequencies, as this will decrease all the elastic scattering rates crucial for interspecies and intraspecies thermalization. In fact, the elastic collision rate for indistinguishable bosons is given by

$$
\gamma=N_{\mathrm{b}} m_{\mathrm{b}} \omega_{\mathrm{b}}^{3} \sigma_{\mathrm{b}} / 2 \pi^{2} k_{\mathrm{B}} T,
$$

where $\sigma_{\mathrm{b}}=8 \pi a_{\mathrm{b}}^{2}$ is the boson elastic cross-section expressed in terms of the boson $s$-wave elastic scattering length $a_{\mathrm{b}}$. The value of $\gamma$ during the cooling strategies described here is shown in the bottom panels of Figure 7 .

If the technical noise and heating sources are properly reduced and the residual background pressure is low enough, the ultimate heating source is set by residual Rayleigh scattering from the laser beams. This depends on various parameters, most notably the laser intensity and the detuning of the laser beam from the atomic resonance, and the corresponding rate reads

$$
\gamma_{i}^{\alpha}(x, y, z)=\frac{\Gamma_{\alpha}^{3}}{8}\left(\frac{\Omega_{i}}{\Omega_{\alpha}}\right)^{3}\left(\frac{1}{\Omega_{\alpha}-\Omega_{i}}+\frac{1}{\Omega_{\alpha}+\Omega_{i}}\right)^{2} \frac{I(x, y, z)}{I_{\alpha}^{\text {sat }}} .
$$



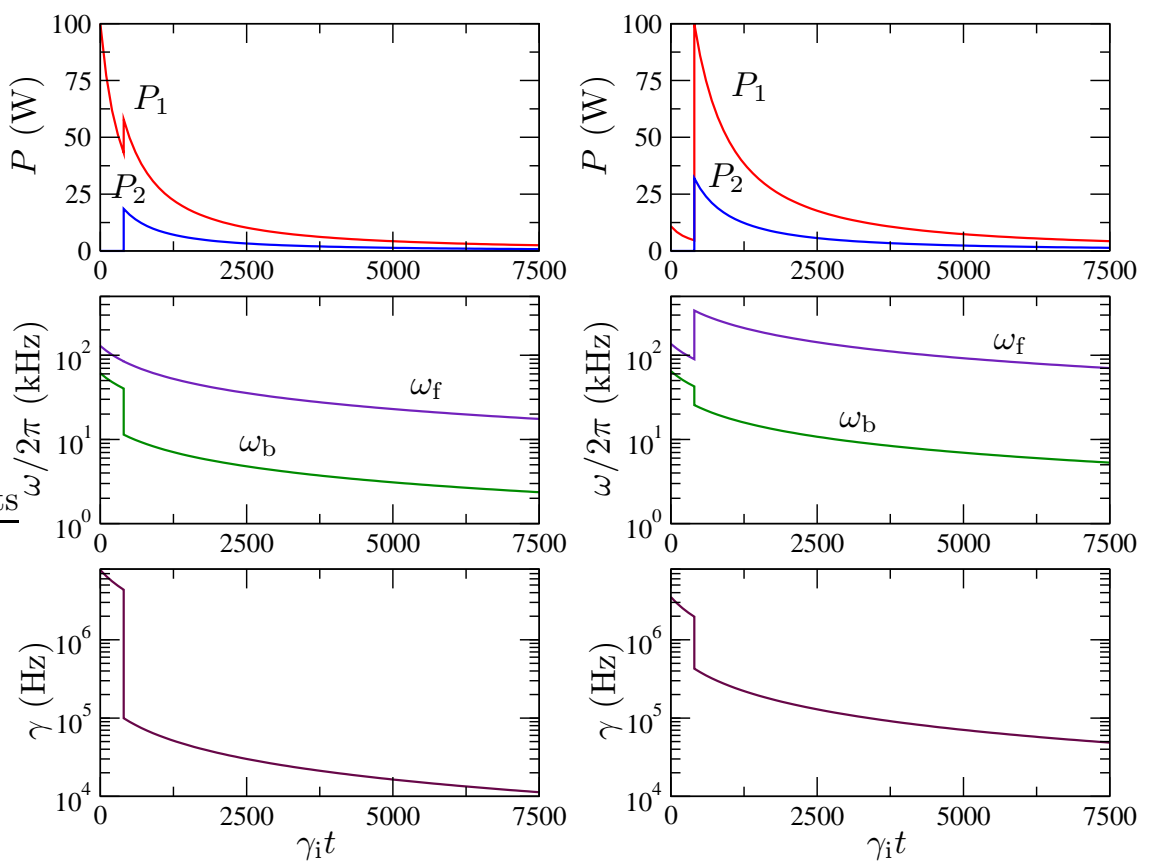

Figure 7: Evaporative cooling strategies in bichromatic optical dipole traps. The time dependence of the laser powers (top panels), the averaged frequencies (center panels), and the bosonic elastic scattering rates (bottom panels), are depicted for single-beam (left) and crossed-beam (right) configurations and a ${ }^{6} \mathrm{Li}^{23} \mathrm{Na}$ mixture. The red-detuned beam is obtained with a Nd:YAG laser emitting at $\lambda_{1}=1064 \mathrm{~nm}$ with a peak power $P_{1}=100 \mathrm{~W}$. For $\gamma_{\mathrm{i}} t>400$, the ratio between the blue- and red-detuned laser powers is kept at the constant value $P_{2} / P_{1}=0.32$. In the case of the single-beam configuration there is a significant decrease in the bosonic trapping frequency, while in the crossed-beam configuration a further gain through the increase of the fermionic trapping frequency is also evident. The large elastic scattering rates available during the entire evaporation process allows for a fast dynamics of thermalization of the Bose gas. We assume equal waists for the beams, $w_{1}=w_{2}=8 \mu \mathrm{m}$. The initial laser power is enough to have $\eta=10$ for a ${ }^{23} \mathrm{Na}$ cloud consisting of $N_{\mathrm{b}}=10^{6}$ atoms transferred from a magneto-optical trap at temperature $T=586 \mu \mathrm{K}$ (crossed-beam) or $T=222 \mu \mathrm{K}$ (single-beam), thermalized with a ${ }^{6} \mathrm{Li}$ cloud with $N_{\mathrm{f}}=10^{5}$ atoms. 


\begin{tabular}{ccccc}
\hline \hline mixture & $\gamma_{1}^{\mathrm{f}}(\mathrm{Hz})$ & $\gamma_{2}^{\mathrm{f}}(\mathrm{Hz})$ & $\gamma_{1}^{\mathrm{b}}(\mathrm{Hz})$ & $\gamma_{2}^{\mathrm{b}}(\mathrm{Hz})$ \\
\hline${ }^{6} \mathrm{Li}^{23} \mathrm{Na}$ & 0.94 & 12.5 & 0.8 & 63 \\
${ }^{6} \mathrm{Li}^{87} \mathrm{Rb}$ & 0.94 & 31 & 4.7 & 306 \\
${ }^{40} \mathrm{~K}^{23} \mathrm{Na}$ & 4.7 & 7.8 & 0.8 & 63 \\
${ }^{40} \mathrm{~K}-{ }^{87} \mathrm{Rb}$ & 4.7 & $9.4 \times 10^{3}$ & 4.7 & $1.1 \times 10^{4}$ \\
\hline \hline
\end{tabular}

Table 3: Estimate of the Rayleigh scattering rates per unit of the corresponding laser power for the fermion-boson mixtures considered in the text. The laser wavelengths are the same as in Table 2 and the beam waists are chosen as $w_{1}=w_{2}=8 \mu \mathrm{m}$.

In the following we will estimate the peak Rayleigh scattering rate, i.e. the value corresponding to the peak laser intensity in the center of the trapping potential, per unit of power of the laser beams in the case of the ${ }^{6} \mathrm{Li}^{2}{ }^{23} \mathrm{Na}$ mixture used in 30. The time dependence of the Rayleigh scattering rate can then be inferred by properly scaling the curves for the laser powers shown in Figure 7. As expected, the strongest source of heating comes from the blue-detuned laser beam acting on the sodium atoms. This is of less concern because the blue-detuned laser beam is turned on only during the latest stage of evaporation, and then raised to a fraction of the reddetuned laser beam. The residual Rayleigh scattering rate is estimated to be around $1.2 \times 10^{-2} \mathrm{~Hz}$, much smaller than the estimated elastic collisional rate, and corresponds to lifetimes well in excess of $10 \mathrm{~s}$. A similar situation occurs also for the ${ }^{40} \mathrm{~K}-{ }^{23} \mathrm{Na}$ mixture. As shown in Table 3, due to the proximity of the atomic transitions for ${ }^{6} \mathrm{Li}$ or ${ }^{40} \mathrm{~K}$ and ${ }^{87} \mathrm{Rb}$ instead, the Rayleigh scattering rates due to the deconfining beam seems prohibitive. However, the large Rayleigh scattering rate per unit of power for the ${ }^{40} \mathrm{~K}$ ${ }^{87} \mathrm{Rb}$ mixture is less frightening since this mixture has also a critical power ratio smaller by one order of magnitude with respect to the other ones, if equal waists are assumed.

The lifetime estimated for ${ }^{6} \mathrm{Li}^{23} \mathrm{Na}$ is long enough to perform various experiments aimed at evidencing superfluidity features, such as mechanical stirring or generation of collective excitations.

\section{Sympathetic cooling and possible evidences for a fermionic superfluid phase}

The study of the dynamics of evaporative cooling is a prerequisite to discuss sympathetic cooling of fermions through their interactions with the Bose gas. Heat exchange between two ensembles is perhaps the most widespread 
thermodynamic process occurring in nature. Thus, as a general method to refrigerate an atomic or molecular ensemble whose direct cooling is difficult to achieve, one can spatially and temporally overlap it with a colder ensemble, the so-called sympathetic cooling. This process has been first demonstrated at the microscopic level for trapped ions [74, then for neutral atoms and molecules via use of cryogenically cooled helium gas [75]. More recently, with the advent of ultracold atomic physics, sympathetic cooling in the microkelvin and nanokelvin ranges has been achieved for bosons in different internal states [76], in different isotopes [77], and for different species 78 .

The elastic scattering properties among fermions and bosons at very low temperatures, which strongly influence the efficacy of sympathetic cooling, are starting to be collected for various atomic mixtures 30, 31. One potential problem common to all the mixtures is the diminished heat exchange capability of bosons when approaching condensation. In particular, fermions can be considered as impurities in the boson cloud and below $T_{\mathrm{c}}$ the Bose gas has a condensed fraction which is expected to be superfluid. Based on the Landau criterion for the critical velocity in a superfluid, we do expect Fermi-Bose collisions to be suppressed when the Fermi velocity becomes smaller than the sound velocity of the Bose gas 26. This has been also experimentally demonstrated [79. The Fermi velocity, defined by $k_{\mathrm{B}} T_{\mathrm{F}}=m_{\mathrm{f}} v_{\mathrm{F}}^{2} / 2$, can be expressed in terms of the trapping parameters as

$$
v_{\mathrm{F}}=1.91\left(\frac{\hbar \omega_{\mathrm{f}}}{m_{\mathrm{f}}}\right)^{1 / 2} N_{\mathrm{f}}^{1 / 6}
$$

On the other hand, the sound velocity $v_{\mathrm{s}}$ for the Bose gas can be written in terms of the chemical potential as $\mu=m_{\mathrm{b}} v_{\mathrm{s}}^{2}$ leading, in the Thomas-Fermi limit, to

$$
v_{\mathrm{s}}=1.22\left(\frac{\hbar^{2} \omega_{\mathrm{b}}^{3} a_{\mathrm{b}} N_{\mathrm{b}}}{m_{\mathrm{b}}^{2}}\right)^{1 / 5}
$$

where $a_{\mathrm{b}}$ is the boson $s$-wave scattering length. The $v_{\mathrm{F}} / v_{\mathrm{s}}$ ratio is, therefore,

$$
\frac{v_{\mathrm{F}}}{v_{\mathrm{s}}}=1.57\left(\frac{\hbar}{m_{\mathrm{b}} \omega_{\mathrm{b}} a_{\mathrm{b}}^{2}}\right)^{1 / 10}\left(\frac{m_{\mathrm{b}}}{m_{\mathrm{f}}}\right)^{1 / 2}\left(\frac{\omega_{\mathrm{f}}}{\omega_{\mathrm{b}}}\right)^{1 / 2} \frac{N_{\mathrm{f}}^{1 / 6}}{N_{\mathrm{b}}^{1 / 5}} .
$$

The following remarks are in order: $a$ ) the velocity ratio (17) scales with the square root of the trapping frequencies, so that it helps to have $\omega_{\mathrm{f}} / \omega_{\mathrm{b}}>>1$ to avoid suppression of Fermi-Bose scattering; $b$ ) the velocity ratio also depends upon the number of fermions and bosons. If the former are kept constant in the trap and the latter undergo evaporative cooling, the velocity ratio is increased; $c$ ) the ratio $v_{\mathrm{F}} / v_{\mathrm{s}}$ is already large for conventional single-color optical dipole traps. For instance, if $\omega_{\mathrm{b}}=2 \pi \times 10^{4}$ 
$\mathrm{s}^{-1}$ and $N_{\mathrm{f}}=N_{\mathrm{b}}$, for a ${ }^{6} \mathrm{Li}^{-23} \mathrm{Na}$ mixture we have $v_{\mathrm{F}} / v_{\mathrm{s}} \simeq 7.2$. In this case, the loss of cooling efficiency becomes relevant for fermion velocities $v \lesssim v_{\mathrm{F}} / 7.2$ corresponding to $T / T_{\mathrm{F}} \lesssim 2 \times 10^{-2}$. It seems therefore that this mixture will hardly enter into the regime where superfluid suppression of impurity scattering is significant.

A more stringent limitation to sympathetic cooling is instead set by the classical heat exchange between bosons and fermions. In Figure 8 we show the dependence of the ratio between the heat capacities for the Bose and the Fermi species, $C_{\mathrm{b}} / C_{\mathrm{f}}$, in bichromatic traps with single and crossedbeam configurations. This gives us a picture of the efficiency of sympathetic cooling since this process breaks down when the ratio $C_{\mathrm{b}} / C_{\mathrm{f}}$ becomes of the order of unity. The heat capacities have been evaluated numerically as described in Appendix A taking into account the time dependence of the various parameters, in particular the diminishing number of bosons during forced evaporation. The effect of many-body interactions on the heat capacity of bosons, evaluated in [80, is next-to-leading with respect to the dependence upon the number of bosons. We see that in the bichromatic traps considered here the heat capacity of the bosons is always larger than that of the fermions by an order of magnitude.

In Figure 8 we also show the temperature ratios $T / T_{\mathrm{F}}$ and $T / T_{\mathrm{c}}$. In both single- and double-beam cases, it is possible to reach $T / T_{\mathrm{F}}<10^{-1}$ while for the Bose gas $T / T_{\mathrm{c}}>5 \times 10^{-1}$, with some slight advantages in the crossed-beam configuration. This means that, with respect to a monochromatic optical dipole trap, a more substantial Bose thermal cloud can be sustained while the Fermi gas is in a deeper degenerate regime. In the various experiments reaching the Fermi degenerate regime, the estimate of the temperature is usually obtained by fitting the surviving normal Bose component. As the temperature is lowered, the thermal component shrinks in amplitude and size, and the fit to assess its temperature is less accurate. This effect is mitigated in our bichromatic traps thus allowing for a more precise thermometry.

Simple estimates for the minimum degeneracy parameter $T^{*} / T_{\mathrm{F}}$ can be obtained by extending the qualitative discussion reported in the concluding part of Section 2. Below $T_{\mathrm{c}}$ the heat capacity of an ideal Bose gas can be written as 81

$$
C_{\mathrm{b}} \simeq 10.8 k_{B} N_{\mathrm{b}}\left(\frac{T}{T_{\mathrm{c}}}\right)^{3}
$$

while for $T / T_{\mathrm{F}} \leq 0.5$ with a good approximation the heat capacity of a Fermi gas is linearly dependent on the temperature (see also Figure 9) 82

$$
C_{\mathrm{f}} \simeq \pi^{2} k_{B} N_{\mathrm{f}} \frac{T}{T_{\mathrm{F}}} .
$$



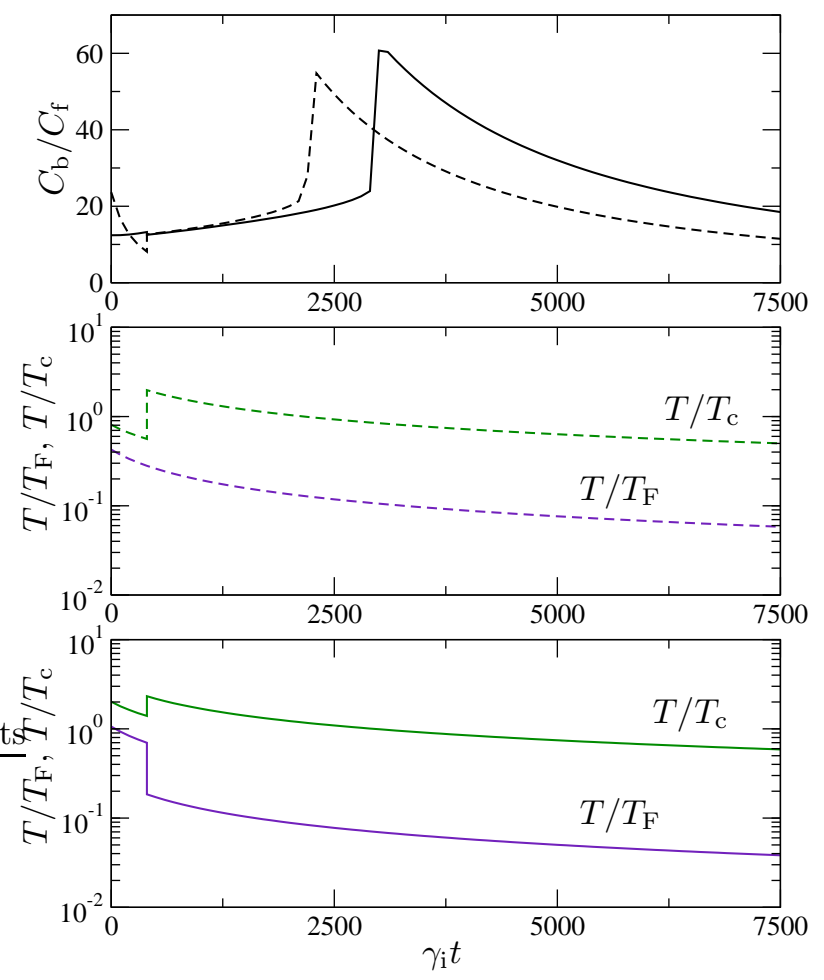

Figure 8: Efficiency for sympathetic cooling of Fermi-Bose mixtures in bichromatic optical dipole traps. In the top panel the ratio between the heat capacities of bosons and fermions $C_{\mathrm{b}} / C_{\mathrm{f}}$ is shown versus time for the evaporative cooling strategies chosen in Figure 7 for single-beam (dashed) and crossed-beam (solid) configurations. The time evolution of the temperature ratios $T / T_{\mathrm{F}}$ and $T / T_{\mathrm{c}}$ is shown for the single-beam (center panel) and crossed-beam (bottom panel) configurations. 
By taking the ratio of $C_{\mathrm{b}}$ and $C_{\mathrm{f}}$ and observing that according to Eqs. (11) and (2) $T_{\mathrm{c}}$ can be expressed in terms of $T_{\mathrm{F}}$, we obtain the degeneracy parameter $T / T_{\mathrm{F}}$ as

$$
\frac{T}{T_{\mathrm{F}}} \simeq 0.35\left(\frac{\omega_{\mathrm{b}}}{\omega_{\mathrm{f}}}\right)^{3 / 2}\left(\frac{C_{\mathrm{b}}}{C_{\mathrm{f}}}\right)^{1 / 2}
$$

In a conservative scenario we can assume that sympathetic cooling stops when $C_{\mathrm{b}} \simeq C_{\mathrm{f}}$. In this case, for $\omega_{\mathrm{f}} / \omega_{\mathrm{b}} \simeq 1$ (as in the case of the ${ }^{6} \mathrm{Li}^{7}{ }^{7} \mathrm{Li}$ mixture) we get a minimum value of the degeneracy parameter at the end of the cooling as $T^{*} / T_{\mathrm{F}} \simeq 0.35$. If cooling is still possible for $C_{\mathrm{b}}<C_{\mathrm{f}}$ more optimistic estimates can be given. For instance if cooling stops when $C_{\mathrm{b}} / C_{\mathrm{f}} \simeq 0.1$, then $T^{*} / T_{\mathrm{F}} \simeq 0.11$. By using a larger $\omega_{\mathrm{f}} / \omega_{\mathrm{b}}$ ratio the $T^{*} / T_{\mathrm{F}}$ ratio decreases according to (20). It is interesting to note that the dependence is quite sensitive to the frequency ratio, and even minor deviations of this ratio from unity result in an observable effect. For instance, in a magnetic trap with a ${ }^{6} \mathrm{Li}^{2}{ }^{23} \mathrm{Na}$ mixture we have $\omega_{\mathrm{f}} / \omega_{\mathrm{b}} \simeq\left(m_{\mathrm{b}} / m_{\mathrm{f}}\right)^{1 / 2} \simeq 1.96$ and therefore we estimate, for $C_{\mathrm{b}} / C_{\mathrm{f}}=0.1$, a value $T^{*} / T_{\mathrm{F}}=0.04$, very close to the minimum value $T / T_{\mathrm{F}}=0.05$ recently obtained by the MIT group for a complete evaporation of the Bose component 35.

Superfluidity of the Fermi gas is expected below the critical temperature for the onset of atomic Cooper pairs [22, 23].

$$
T_{\mathrm{BCS}}=\frac{5}{3 e} e^{-\pi / 2 k_{\mathrm{F}}\left|a_{\mathrm{f}}\right|} T_{\mathrm{F}},
$$

where $k_{\mathrm{F}}$ is the Fermi wavevector such that $E_{\mathrm{F}}=\hbar^{2} k_{\mathrm{F}}^{2} / 2 m_{\mathrm{f}}$, and $a_{\mathrm{f}}$ is the fermion-fermion elastic scattering length. Besides leaving freedom to apply arbitrary homogeneous magnetic fields to enhance the scattering length through tuning to a Feshbach resonance [44, 45, 47, as demonstrated experimentally for Fermi gases in [56, 57, our bichromatic configuration allows also for an independent increase of $k_{\mathrm{F}}$ due to the higher achievable densities. The resulting $T_{\mathrm{BCS}} / T_{\mathrm{F}}$ are within the accessible range which corresponds, as seen in Figure 8 , to $T / T_{\mathrm{F}} \gtrsim 3 \times 10^{-2}$. The use of optical trapping also leads to a large absolute value of the Fermi temperature which can be otherwise obtained by magnetic trapping only with particular geometries maximizing the field gradients 83. Finally, the presence of the Bose gas could allow for enhancements of the BCS pairing temperature since bosons can mediate phonon-exchange between fermions in a way analogous to ordinary superconductors 84, 85.

In general, with the technique discussed above new mixtures consisting of a normal Bose gas (or a Bose condensed gas coexisting with a large Bose thermal fraction) and a degenerate Fermi gas are viable. This contrasts the only situation known so far of a ${ }^{3} \mathrm{He}-{ }^{4} \mathrm{He}$ mixture where degeneracy 
is reached earlier for the bosonic species. One of the advantages of using such an anomalous mixture is the possibility to have a well controllable background - a normal Bose gas - superimposed to the Fermi gas. This considerably simplifies the possible signatures of a superfluid phase transition in a Fermi gas. For instance it should possible to look at a bulge in the density distribution as predicted in [46, since this is obtained admist a smooth, low density and well controllable thermal cloud instead of a higher density and peaked condensate [86]. The presence of a superfluid state could be evidenced also by using the same blue-detuned beam as a mechanical stirrer for the fermion cloud. In this case one should look at a finite threshold for the onset of a dissipative motion or a drag force [17, 18]. The presence of the bosonic thermal cloud (or a condensate component) gives rise to heating for all stirring velocities (or above a critical velocity depending upon the condensate density) of the laser beam [87. However the contribution of the thermal cloud to the heating is very low, and much smaller than the Rayleigh scattering in the relevant range of stirring velocities, due to the low density. In order to better discriminate heating coming from the Bose component, one could also take advantage of the proposed manipulation of an ultracold cloud with Raman beams [88, 89] to create a directional critical velocity for the Fermi component. Another advantage of a Fermi-Bose mixture with the latter component in the normal state is the possibility to perform experiments on scattering from microscopic impurities (such as the one described in $[79$ for a Bose condensate) in a much simpler way than using two isotopes.

The presence of the Bose cooler in the non-condensed phase allows one to re-examine also species for which Bose condensation has been proven difficult to achieve, like cesium (see however the recent achievement of BEC in Cs with purely optical means [55]). For instance, one can reconsider the use of ${ }^{133} \mathrm{Cs}$ [90] which, due to its large mass and small recoil temperature, can be efficiently cooled to very low temperature in a magneto-optical trap. This would ensure robust initial conditions, in terms of initial number of atoms and initial temperature, to start an efficient evaporative cooling in the optical dipole trap. Even if sympathetic cooling is less efficient due to the large mass ratio with the Fermi species 91, the same feature is an advantage in terms of ratio between the trapping frequencies even at zero blue-detuned beam intensity or in a purely magnetic trap. Recently, sympathetic cooling of ${ }^{7} \mathrm{Li}$ through ${ }^{133} \mathrm{Cs}$ has been demonstrated in a far-off resonance optical trap 92. $\mathrm{A} \mathrm{CO}_{2}$ laser was used to create the optical potential obtaining, due to the static atomic polarizabilities, a larger energy depth for ${ }^{133} \mathrm{Cs}$. As a consequence the ${ }^{7} \mathrm{Li}$ went under simultaneous sympathetic and evaporative cooling. The problem can be circumvented by using a Nd:YAG laser as the primary, red-detuned trapping laser, and a deconfining beam with wavelength in between the two atomic transition 
wavelengths. Furthermore, by relaxing the requirement for using an alkali species (using for instance ytterbium cooled in an optical dipole trap, see 93 94, and recently brought into the degenerate regime for a bosonic isotope [95]) various favourable possibilities can be envisaged.

\section{Conclusions}

A novel path to reach deep Fermi degeneracy through sympathetic cooling with a Bose gas undergoing evaporative cooling in an optical dipole trap has been proposed. The key feature is that the trapping frequencies, determining the degree of degeneracy of the dilute gases, are made different by properly using a second, deconfining beam. Both single- and crossed-beam configurations have been studied for the four Fermi-Bose mixtures available with stable alkali fermions. In all cases, a substantial increase of the Fermi energy is expected maintaining at the same time the critical temperature for Bose condensation constant or slightly decreased with respect to a single color optical dipole trap. This decrease of the critical temperature for Bose condensation is beneficial for maintaining a precision thermometry in the deep degenerate regime for the Fermi gas, and it makes also possible the use of species which can hardly reach Bose condensation. With respect to the routes for fermion cooling currently under active investigation, our proposal does not necessarily require enhancement of elastic scattering lengths through Feshbach resonances, a mechanism often associated to the increase of inelastic processes rates, in competition with hydrodynamic behaviour of the atomic clouds or formation of ultracold molecules 33, 34, 35, 36, already present even in nondegenerate conditions [96]. With respect to the pure Fermi mixtures, it allows instead for a more precise determination of the temperature and, in particular, of the BCS-like phase transition temperature below which onset of superfluidity is expected [23]. Two technical issues still to be addressed in detail are the degree of control of the laser beams to achieve a common focus, and the intensity ratio stability between confining and deconfining lasers. In a more pessimistic scenario, the proposed technique will allow to study the heating mechanisms preventing further cooling of fermions, in a way similar to the one proposed in 97, or non-equilibrium phenomena in the ultracold regime analogous to those already explored for the Bose condensed gases [98, or proposed as an interesting alternative to quasi-equilibrium sympathetic cooling [99].

\section{Acknowledgments}

We are indebted to our teacher, colleague, and friend Giovanni Jona-Lasinio for longstanding, pleasant scientific discussions and for having encouraged 
us in pursuing independent thinking at an early stage of our carrier. This research was partially supported by Cofinanziamento MIUR protocollo 2002027798. R. O. also acknowledges support by the Department of Energy, under Contract No. W-7405-ENG-36.

\section{A Ideal Bose and Fermi systems with a finite number of particles in a harmonic poten- tial}

In this section we review some elementary thermodynamic properties of noninteracting quantum systems consisting of a finite number of bosonic or fermionic particles trapped into an external potential. More specifically, we suppose that the particles of mass $m$ are confined by the anisotropic harmonic potential

$$
V(x, y, z)=\frac{1}{2} m\left(\omega_{x}^{2} x^{2}+\omega_{y}^{2} y^{2}+\omega_{z}^{2} z^{2}\right),
$$

so that the corresponding single-particle energy levels are given by

$$
E_{n_{x}, n_{y}, n_{z}}=\hbar \omega_{x}\left(n_{x}+\frac{1}{2}\right)+\hbar \omega_{y}\left(n_{y}+\frac{1}{2}\right)+\hbar \omega_{z}\left(n_{z}+\frac{1}{2}\right),
$$

with $n_{x}, n_{y}, n_{z}=0,1,2, \ldots$.

In the grand-canonical ensemble, the average number of particles and the energy of the system are, respectively

$$
\begin{aligned}
N & =\sum_{n_{x}=0}^{\infty} \sum_{n_{y}=0}^{\infty} \sum_{n_{z}=0}^{\infty} \frac{1}{\exp \left(\frac{E_{n_{x}, n_{y}, n_{z}}-\mu}{k_{\mathrm{B}} T}\right) \pm 1} \\
E & =\sum_{n_{x}=0}^{\infty} \sum_{n_{y}=0}^{\infty} \sum_{n_{z}=0}^{\infty} \frac{E_{n_{x}, n_{y}, n_{z}}}{\exp \left(\frac{E_{n_{x}, n_{y}, n_{z}}-\mu}{k_{\mathrm{B}} T}\right) \pm 1},
\end{aligned}
$$

where $T$ is the temperature of the reservoir, $\mu$ the chemical potential, and the upper (lower) sign holds for fermions (bosons). Usually, one knows the temperature $T$ of the reservoir and the number $N$ of particles of the system so that the chemical potential must be evaluated as a function of $N$ and $T$. The value of $\mu(N, T)$ is determined by solving the nonlinear Eq. (24) numerically, e.g. by truncating the series in the r.h.s. at a proper order and controlling the convergence error [100]. Once $\mu(N, T)$ is known, the energy $E(\mu(N, T), T)$ is evaluated in an analogous way by truncating the series in the r.h.s. of (25). 
The computation of the series in (24) or (25) requires the evaluation of triply nested sums which may be very time consuming. A more favourable situation, with double or single series to be computed, is obtained in the presence of symmetries of the external potential. For instance, in the case of experimental interest in which $\omega_{x}=\omega_{y} \equiv \omega_{x y}$, Eq. (24) becomes

$$
N=\sum_{n_{x y}=0}^{\infty} \sum_{n_{z}=0}^{\infty} \frac{n_{x y}+1}{\exp \left(\frac{E_{n_{x y}, n_{z}}-\mu}{k_{\mathrm{B}} T}\right) \pm 1},
$$

where the factor $n_{x y}+1$ represents the degeneracy of the level at energy $E_{n_{x y}, n_{z}}=\hbar \omega_{x y}\left(n_{x y}+1\right)+\hbar \omega_{z}\left(n_{z}+\frac{1}{2}\right)$ with $n_{x y}, n_{z}=0,1,2, \ldots$

The heat capacity of the system at fixed number of particles is

$$
\begin{aligned}
C(N, T) & =\frac{\mathrm{d} E(N, T)}{\mathrm{d} T} \\
& =\frac{\partial E}{\partial T}+\frac{\partial E}{\partial \mu} \frac{\partial \mu}{\partial T} \\
& =\frac{\partial E}{\partial T}-\frac{\partial E}{\partial \mu} \frac{\partial N}{\partial T}\left(\frac{\partial N}{\partial \mu}\right)^{-1}
\end{aligned}
$$

where we used the constraint $\mathrm{d} N / \mathrm{d} T=\partial N / \partial T+(\partial N / \partial \mu)(\partial \mu / \partial T)=0$. The derivatives $\partial N / \partial T, \partial N / \partial \mu, \partial E / \partial T$, and $\partial E / \partial \mu$ are obtained by (24) and (25) and can be evaluated numerically as explained above. As an example, in Figure 9 we show the behavior of $C(N, T)$ as a function of $T$ for different values of the number of particles $N$. While in the fermion case $C(N, T)$ is almost independent of $N$, for bosons the size effect is striking around the critical temperature.

Finally, we derive the values of the Fermi and Bose degeneracy temperatures reported in (11) and (2). In the case of fermions, for any value of $N$ and $T$ we have $\mu(N, T)<\mu(N, T=0)$ and the Fermi energy is defined as $E_{\mathrm{F}}(N)=\mu(N, T=0)$. For $k_{\mathrm{B}} T \gg \hbar \omega$, a condition well fulfilled in the experiments, the Fermi energy can be evaluated with the approximation

$$
\begin{aligned}
N & =\sum_{\substack{n_{x}, n_{y}, n_{z} \\
E_{n x, n y, n z}<E_{\mathrm{F}}}} 1 \\
& \simeq \frac{\frac{1}{6} E_{\mathrm{F}}^{3}}{\hbar \omega_{x} \hbar \omega_{y} \hbar \omega_{z}}=\frac{E_{\mathrm{F}}^{3}}{6 \hbar^{3} \omega_{\mathrm{f}}^{3}},
\end{aligned}
$$

where $\omega_{\mathrm{f}}=\left(\omega_{x} \omega_{y} \omega_{z}\right)^{1 / 3}$. Therefore, $k_{\mathrm{B}} T_{\mathrm{F}} \equiv E_{\mathrm{F}}=(6 N)^{1 / 3} \hbar \omega_{\mathrm{f}}$.

In the case of bosons, we separate the occupation of the ground state 

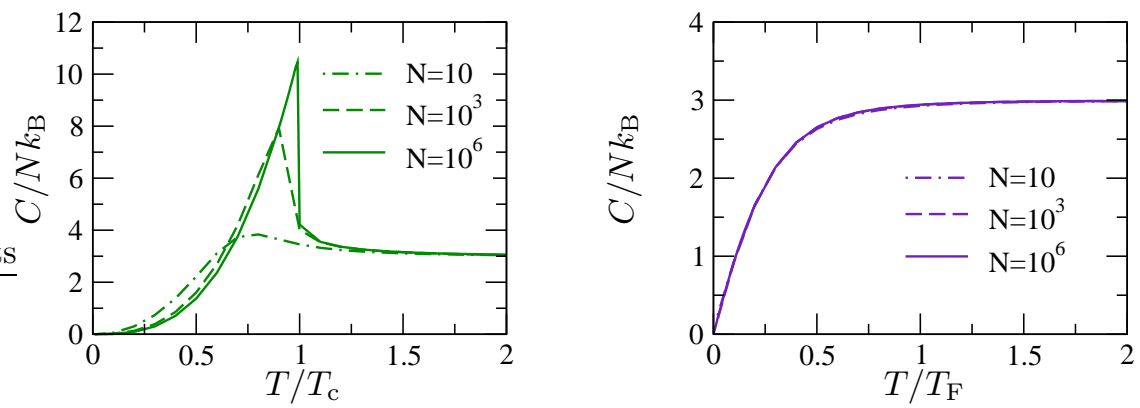

Figure 9: $\quad$ Specific heats of a system of $N$ noninteracting bosons (left panel) and fermions (right panel) in a harmonic trap as a function of the normalized temperatures $T / T_{\mathrm{c}}$ and $T / T_{\mathrm{F}}$. We have chosen $\omega_{x}=\omega_{y}=$ $\omega_{z} / \sqrt{2}$ as in a crossed-beam optical dipole trap.

from that of the excited ones:

$$
\begin{aligned}
N & =N_{0}+N_{e} \\
& =\frac{1}{e^{\frac{E_{0}-\mu(N, T)}{k_{\mathrm{B}} T}}-1}+\sum_{\substack{n_{x}, n_{y}, n_{z} \\
\neq 0,0,0}} \frac{1}{e^{\frac{E_{n_{x}, n_{y}, n_{z}-\mu(N, T)}}{k_{\mathrm{B}} T}}-1},
\end{aligned}
$$

where $\mu(N, T)<E_{0} \equiv E_{0,0,0}$. When $T \rightarrow 0$, we have $\mu \rightarrow E_{0}$ and $N_{0} \rightarrow N$. The critical temperature $T_{\mathrm{c}}$ is defined by the condition $N_{e}\left(\mu=E_{0}, T_{\mathrm{c}}\right)=$ $N$. For $k_{\mathrm{B}} T \gg \hbar \omega$, we have

$$
\begin{aligned}
N_{e}\left(\mu=E_{0}, T_{\mathrm{c}}\right) & \simeq \int_{0}^{\infty} \mathrm{d} n_{x} \mathrm{~d} n_{y} \mathrm{~d} n_{z} \frac{1}{e^{\frac{n_{x} \hbar \omega_{x}+n_{y} \hbar \omega_{y}+n_{z} \hbar \omega_{z}}{k_{\mathrm{B}} T_{\mathrm{c}}}}-1} \\
& =\left(\frac{k_{\mathrm{B}} T_{\mathrm{c}}}{\hbar \omega_{\mathrm{b}}}\right)^{3} \int_{0}^{\infty} \mathrm{d} x \mathrm{~d} y \mathrm{~d} z \frac{1}{e^{x+y+z}-1} \\
& =\left(\frac{k_{\mathrm{B}} T_{\mathrm{c}}}{\hbar \omega_{\mathrm{b}}}\right)^{3} \zeta(3),
\end{aligned}
$$

where $\omega_{\mathrm{b}}=\left(\omega_{x} \omega_{y} \omega_{z}\right)^{1 / 3}$ and $\zeta$ is the Riemann zeta function. Therefore, $k_{\mathrm{B}} T_{\mathrm{c}}=(N / \zeta(3))^{1 / 3} \hbar \omega_{\mathrm{b}}$.

\section{References}

[1] Y. Nambu and G. Jona-Lasinio, Dynamical model of elementary particles based on an analogy with superconductivity. I, Phys. Rev. 122: 345 (1961); II, 124: 246 (1961). 
[2] D. R. Tilley and J. Tilley, Superfluidity and Superconductivity, (Institute of Physics, Bristol and Philadelphia, 1996).

[3] S. Chu, The manipulation of neutral particles, Rev. Mod. Phys. 70: 685 (1998).

[4] C. N. Cohen-Tannouji, Manipulating atoms with photons, Rev. Mod. Phys. 70: 707 (1998).

[5] W. D. Phillips, Laser cooling and trapping of neutral atoms, Rev. Mod. Phys. 70: 721 (1998).

[6] W. Ketterle and N. J. Van Druten, Evaporative cooling of trapped atoms, in Advances in Atomic, Molecular, and Optical Physics, edited by B. Bederson and H. Walther, Vol. 37 (Academic Press, San Diego, 1996), p. 181.

[7] M. H. Anderson, J. R. Ensher, M. R. Matthews, C. E. Wieman, and E. A. Cornell, Observation of Bose-Einstein condensation in a dilute atomic vapor, Science 269: 198 (1995).

[8] K. B. Davis, M.-O. Mewes, M. R. Andrews, N. J. van Druten, D. S. Durfee, D. M. Kurn, and W. Ketterle, Bose-Einstein condensation in a gas of sodium atoms, Phys. Rev. Lett. 75: 3969 (1995).

[9] C. C. Bradley, C. A. Sackett, J. J. Tollett, and R. G. Hulet, Evidence of Bose-Einstein condensation in an atomic gas with attractive interactions, Phys. Rev. Lett. 75: 1687 (1995); ibidem 79: 1170 (1997).

[10] Bose-Einstein condensates and atom lasers, C. R. Acad. Sci. Paris, t. 2, Sèrie IV, no. 3 (2001), Special issue edited by A. Aspect and J. Dalibard.

[11] C. J. Pethick and H. Smith, Bose-Einstein condensation in dilute gases, (Cambridge University Press, Cambridge, 2002).

[12] L. P. Pitaevskii and S. Stringari, Bose-Einstein condensation, (Oxford Science Publications, Oxford, 2003).

[13] M. R. Matthews, B. P. Anderson, P. C. Kaljan, D. S. Hall, C. E. Wieman, and E. A. Cornell, Vortices in a Bose-Einstein condensate, Phys. Rev. Lett. 83: 2498 (1999).

[14] K. W. Madison, F. Chevy, W. Wohlleben, and J. Dalibard, Vortex formation in a stirred Bose-Einstein condensate, Phys. Rev. Lett. 84: 806 (2000). 
[15] J. R. Abo-Shaeer, C. Raman, J. M. Vogels, and W. Ketterle, Observation of vortex lattices in Bose-Einstein condensates, Science 292: 476 (2001).

[16] O. M. Maragò, S. A. Hopkins, J. Arlt, E. Hodby, G. Hechenblaikner, and C. J. Foot, Observation of the scissor mode and evidence for superfluidity of a trapped Bose-Einstein condensed gas, Phys. Rev. Lett. 84: 2059 (2000).

[17] C. Raman, M. Köhl, R. Onofrio, D. S. Durfee, C. E. Kuklewicz, Z. Hadzibabic, and W. Ketterle, Evidence for a critical velocity in a BoseEinstein condensed gas, Phys. Rev. Lett. 84: 2502 (1999).

[18] R. Onofrio, C. Raman, J. M. Vogels, J. Abo-Shaeer, A. P. Chikkatur, and W. Ketterle, Observation of a superfluid flow in a Bose-Einstein condensed gas, Phys. Rev. Lett. 85: 2228 (2000).

[19] A. L. Fetter and A. A. Svidzinsky, Vortices in a trapped dilute BoseEinstein condensate, J. Phys. Condens. Matter 13: R135 (2001).

[20] A. J. Leggett, Bose-Einstein condensation in the alkali gases: Some fundamental concepts, Rev. Mod. Phys. 73: 307 (2001).

[21] L. P. Pitaevskii and S. Stringari, The quest for superfluidity in Fermi gases, Science 298: 2144 (2001).

[22] L. P. Gor'kov and T. K. Melik-Barkhudarov, Contribution to the theory of superfluidity in an imperfect Fermi gas, Zh. Eksp. Teor. Fiz 40: 1452 (1961) [Sov. Phys. JETP 13: 1018 (1961)].

[23] H. T. C. Stoof, M. Houbiers, C. A. Sackett, and R. G. Hulet, Superfluidity of spin-polarized Li-6, Phys. Rev. Lett. 76: 10 (1996).

[24] R. Onofrio and C. Presilla, Reaching Fermi degeneracy in two-species optical dipole traps, Phys. Rev. Lett. 89: 100401 (2002).

[25] B. DeMarco and D. S. Jin, Onset of Fermi degeneracy in a trapped atomic gas, Science 285: 1703 (1999).

[26] E. Timmermans and R. Cotè, Superfluidity in sympathetic cooling with atomic Bose-Einstein condensates, Phys. Rev. Lett. 80: 3419 (1998).

[27] A. G. Truscott, K. E Strecker, W. I. McAlexander, G. B. Partridge, and R. G. Hulet, Observation of Fermi pressure in a gas of trapped atoms, Science 291: 2570 (2001). 
[28] F. Schreck, L. Khaykovich, K. L. Corwin, G. Ferrari, T. Bourdel, J. Cubizolles, and C. Salomon, Quasipure Bose-Einstein condensate immersed in a Fermi sea, Phys. Rev. Lett. 87: 080403 (2001).

[29] S. R. Granade, M. E. Gehm, K. M. O'Hara, and J. E. Thomas, Alloptical production of a degenerate Fermi gas, Phys. Rev. Lett. 88: 120405 (2002).

[30] Z. Hadzibabic, C. A. Stan, K. Dieckmann, S. Gupta, M. W. Zwierlein, A. Görlitz, and W. Ketterle, Two-species mixture of quantum degenerate Bose and Fermi gases, Phys. Rev. Lett. 88: 160401 (2002).

[31] G. Roati, F. Riboli, G. Modugno, and M. Inguscio, Fermi-Bose quantum degenerate ${ }^{40} \mathrm{~K}-{ }^{87} \mathrm{Rb}$ mixture with attractive interaction, Phys. Rev. Lett. 89: 150403 (2002).

[32] M. E. Gehm, S. L. Hemmer, S. R. Granade, K. M. O'Hara, and J. E. Thomas, Mechanical stability of a strongly interacting Fermi gas of atoms, Phys. Rev. A 68: 011401(R) (2003).

[33] C. A. Regal, C. Tickner, J. L. Bohn, and D. S. Jin, Creation of ultracold molecules from a Fermi gas of atoms, Nature 424: 47 (2003).

[34] K. E. Strecker, G. B. Partridge, and R. G. Hulet, Conversion of an atomic Fermi gas to a long-lived molecular Bose gas, Phys. Rev. Lett. 91: 080406 (2003).

[35] Z. Hadzibabic, S. Gupta, C. A. Stan, C. H. Schunck, M. W. Zwierlein, K. Dieckmann, and W. Ketterle, Phys. Rev. Lett. 91: 160401 (2003).

[36] J. Cubizolles, T. Bourdel, S.J.J.M.F. Kokkelmans, G. V. Shlyapnikov, and C. Salomon, Phys. Rev. Lett. 91: 240401 (2003).

[37] J. Goldwin, S. B. Papp, B. DeMarco, and D. S. Jin, Two-species magneto-optical trap with ${ }^{40} \mathrm{~K}$ and ${ }^{87} \mathrm{Rb}$, Phys. Rev. A 65: 021402 (2001).

[38] H. Feshbach, A unified theory of nuclear reactions II, Ann. Phys. (NY) 19: 287 (1962).

[39] E. Timmermans, P. Tommasini, M. Hussein, and A. Kerman, Feshbach resonances in atomic Bose-Einstein condensates, Phys. Rep. 315: 199 (1999).

[40] S. Inouye, M. R. Andrews, J Stenger, H.J. Miesner, D. M. StamperKurn, and W. Ketterle, Observation of Feshbach resonances in a BoseEinstein condensate, Nature 392: 151 (1998). 
[41] Ph. Courteille, R. S. Freeland, D.J. Heinzen, F. A. van Abeelen, B. J. Verhaar, Observation of a Feshbach resonance in cold atom scattering, Phys. Rev. Lett. 81: 69 (1998).

[42] J. L. Roberts, N. R. Claussen, J. P. Burke, Jr., C. H. Greene, E. A. Cornell, and C. E. Wieman, Resonant magnetic field control of elastic scattering in cold ${ }^{85} \mathrm{Rb}$, Phys. Rev. Lett. 81: 5109 (1998).

[43] V. Vuletic, A. J. Kerman, C. Chin, S. Chu, Observation of low-field Feshbach resonances in collision of Cesium atoms, Phys. Rev. Lett. 82: 1406 (1999).

[44] E. Timmermans, V. Furuya, P. W. Milonni, and A. K. Kerman, Prospect of creating a composite Fermi-Bose superfluid, Phys. Lett. A 285: 228 (2001).

[45] M. Holland, S.J.J.M.F. Kokkelmans, M. L. Chiofalo, and R. Walser, Resonance superfluidity in a quantum degenerate gas, Phys. Rev. Lett. 87: 120406 (2001).

[46] M. L. Chiofalo, S.J.J.M.F. Kokkelmans, J. N. Milstein, and M. J. Holland, Signatures of resonance superfluidity in a quantum Fermi gas, Phys. Rev. Lett. 88: 120406 (2002).

[47] Y. Ohashi and A. Griffin, BCS-BEC crossover in a gas of Fermi atoms with a Feshbach resonance, Phys. Rev. Lett. 89: 130402 (2002).

[48] A. Askin and J. Gordon, Cooling and trapping of atoms by resonance radiation pressure, Opt. Lett. 4: 161 (1979).

[49] J. P. Gordon and A. Askin, Motion of atoms in a radiation trap, Phys. Rev. A 21: 1606 (1980).

[50] S. Chu, J. E. Bjorkholm, A. Askin, and A. Cable, Experimental observation of optically trapped atoms, Phys. Rev. Lett. 57: 314 (1986).

[51] J. Miller, R. Cline, and D. Heinzen, Far-off resonance optical trapping of atoms, Phys. Rev. A 47: R4567 (1993).

[52] C. S. Adams, H. J. Lee, N. Davidson, M. Kasevich, and S. Chu, Evaporative cooling in a crossed dipole trap, Phys. Rev. Lett. 74: 3577 (1995).

[53] D. M. Stamper-Kurn, M. R. Andrews, A. P. Chikkatur, S. Inouye, H.-J. Miesner, J. Stenger, and W. Ketterle, Optical confinement of a Bose-Einstein condensate, Phys. Rev. Lett. 80: 2027 (1998). 
[54] M. D. Barrett, J. A. Sauer, and M. S. Chapman, All-optical formation of an atomic Bose-Einstein condensate, Phys. Rev. Lett. 87: 010404 (2001).

[55] R. Grimm, M. Weidemüller, and Y. B. Ovchinkov, Optical dipole traps for neutral atoms, Ad. At. Mol. Op. 42: 95 (2000).

[56] T. Loftus, C. A. Regal, C. Tickner, J. L. Bohn, and D. S. Jin, Resonant control of elastic collisions in an optically trapped Fermi gas of atoms, Phys. Rev. Lett. 88: 173201 (2002).

[57] G. Modugno, G. Roati, F. Riboli, F. Ferlaino, R. J. Brecha, and M. Inguscio, Collapse of a degenerate Fermi gas, Science 297: 2240 (2002).

[58] K. M. O'Hara, S. L. Hemmer, M. E. Gehm, S. R. Granade, J. E. Thomas, Observation of a strongly interacting degenerate Fermi gas of atoms, Science 298: 2179 (2002).

[59] In this experiment the lowest $T / T_{\mathrm{F}}$ ratio with dual evaporative cooling of two hyperfine fermion states has been achieved, as $0.08<T / T_{\mathrm{F}}<$ 0.18. By performing an identical evaporation process on an equally populated mixture of the two hyperfine states the heat capacities are always well matched. On the other hand any determination of the temperature for a system made of degenerate fermions is strongly model dependent and drops in sensitivity at the lowest explored temperatures, as witnessed by the relatively large range of values for the estimated $T / T_{\mathrm{F}}$ ratio.

[60] C. Menotti, P. Pedri, and S. Stringari, Expansion of an interacting Fermi gas, Phys. Rev. Lett. 89: 250402 (2002).

[61] C. Presilla and R. Onofrio, Cooling dynamics of ultracold two-species Fermi-Bose mixtures, Phys. Rev. Lett. 90: 030404 (2003).

[62] L. Viverit, S. Giorgini, L. P. Pitaevskii, and S. Stringari, Adiabatic compression of a trapped Fermi gas, Phys. Rev. A 63: 033603 (2001).

[63] N. R. Thomas, A. C. Wilson, C. J. Foot, Double-well magnetic trap for Bose-Einstein condensation, Phys. Rev. A 65: 063406 (2002).

[64] U. Schünemann, H. Engler, M. Zielonkowski, M. Weidemüller, and R. Grimm, Magneto-optic trapping of lithium using semiconductor lasers, Optics Communications 158: 263 (1998).

[65] G. Modugno, C. Benkö, P. Hannaford, G. Roati, and M. Inguscio, Sub-Doppler laser cooling of fermionic ${ }^{40} \mathrm{~K}$ atoms, Phys. Rev. A 60: R3373 (1999). 
[66] T. Takekoshi, J. R. Yeh, R. J. Knize, Quasi-electrostatic trap for neutral atoms, Optics Communications 114: 421 (1995).

[67] T. Takekoshi and R. J. Knize, $\mathrm{CO}_{2}$ laser trap for cesium atoms, Optics Letters 21: 77 (1996).

[68] K. M. O'Hara, S. R. Granade, M. E. Gehm, T. A. Savard, S. Bali, C. Freed, and J. E. Thomas, Ultrastable $\mathrm{CO}_{2}$ laser trapping of lithium atoms, Phys. Rev. Lett. 82: 4204 (1999).

[69] K. M. O'Hara, S. R. Granade, M. E. Gehm, and J. E. Thomas, Loading dynamics of $\mathrm{CO}_{2}$ laser traps, Phys. Rev. A 63: 043403 (2001).

[70] T.A. Savard, K.M. O'Hara, and J. E. Thomas, Laser-noise-induced heating in far-off resonance optical traps, Phys. Rev. A 56: R1095 (1997).

[71] K. M. O'Hara, M. E. Gehm, S.R. Granade, and J. E. Thomas, Scaling laws for evaporative cooling in time-dependent optical traps, Phys. Rev. A 64: 051403R (2001).

[72] M. Houbiers, H. T. C. Stoof, W. I. McAlexander, and R. G. Hulet, Elastic and inelastic collisions of Li-6 atoms in magnetic and optical traps, Phys. Rev. A 57: R1497 (1998).

[73] In the case of magnetic trapping for alkali species confinement and evaporation are instead kept separated. It is then possible to further compress the atomic cloud during rf-induced evaporation, which explains the earlier success of this technique to achieve the Bosedegenerate regime.

[74] D. J. Larson, J. C. Bergquist, J. J. Bollinger, W. M. Itano, and D. J. Wineland, Sympathetic cooling of trapped ions: A laser-cooled twospecies nonneutral ion plasma, Phys. Rev. Lett. 57: 70 (1986).

[75] R. deCarvalho, J. M. Doyle, B. Friedrich, T. Guillet, J. Kim, D. Patterson, and J. D. Weinstein, Buffer-gas loaded magnetic traps for atoms and molecules: A primer, Eur. Phys. J. D 7: 289 (1999).

[76] C. Myatt, E. Burt, R. Ghrist, E. Cornell, and C. Wieman, Production of two overlapping Bose-Einstein condensates by sympathetic cooling, Phys. Rev. Lett. 78: 586 (1997).

[77] I. Bloch, M. Greiner, O. Mandel, T. W. Hänsch, and T. Esslinger, Sympathetic cooling of ${ }^{85} \mathrm{Rb}$ and ${ }^{87} \mathrm{Rb}$, Phys. Rev. A 64: 021402(R) (2001). 
[78] G. Modugno, G. Ferrari, G. Roati, A. Simoni, and M. Inguscio, BoseEinstein condensation of potassium atoms by sympathetic cooling, Science 294: 1320 (2001).

[79] A. Chikkatur, A. Görlitz, D. M. Stamper-Kurn, S. Inouye, S. Gupta, and W. Ketterle, Suppression and enhancement of impurity scattering in a Bose-Einstein condensate, Phys. Rev. Lett. 85: 483 (2000).

[80] S. Giorgini, L. P. Pitaevskii, and S. Stringari, Thermodynamics of a trapped Bose-condensed gas, J. Low Temp. Phys. 109: 309 (1997).

[81] V. Bagnato, D. E. Pritchard, and D. Kleppner, Bose-Einstein condensation in an external potential, Phys. Rev. A 35: 4354 (1987).

[82] D. A. Butts and D. S. Rokhsar, Trapped Fermi gases, Phys. Rev. A 55: 4346 (1997).

[83] F. Schreck, G. Ferrari, K. L. Corwin, J. Cubizolles, L. Khaykovich, M.-O. Mewes, and C. Salomon, Sympathetic cooling of bosonic and fermionic lithium gases towards quantum degeneracy, Phys. Rev. A 64: 011402(R) (2001).

[84] H. Heiselberg, C. J. Pethick, H. Smith, and L. Viverit, Influence of induced interaction on the superfluid transition in dilute Fermi gases, Phys. Rev. Lett. 85: 2418 (2000).

[85] M. J. Bijlsma, B. A. Heringa, and H.T.C. Stoof, Phonon exchange in dilute Fermi-Bose mixtures: Tailoring the Fermi-Fermi interaction, Phys. Rev. A 61: 053601 (2000).

[86] M. Amoruso, A. Minguzzi, S. Stringari, M. P. Tosi, and L. Vichi, Temperature-dependent density profiles of trapped boson-fermion mixtures, Eur. Phys. J. D 4: 261 (1998).

[87] C. Raman, R. Onofrio, J. M. Vogels, J. R. Abo-Shaeer, and W. Ketterle, Dissipationless flow and superfluidity in gaseous Bose-Einstein condensates, J. Low Temp. Phys. 122: 99 (2001).

[88] J. Higbie and D.M. Stamper-Kurn, Periodically dressed Bose-Einstein condensate: A superfluid with an anisotropic and variable critical velocity, Phys. Rev. Lett. 88: 090401 (2002).

[89] D. M. Stamper-Kurn, Anisotropic dissipation of superfluid flow in a periodically dressed Boes-Einstein condensate, New J. Phys. 5: 50 (2003). 
[90] D. Boiron, A. Michaud, J. M. Fournier, L. Simard, M. Sprenger, G. Grynberg, and C. Salomon, Cold and dense cesium clouds in fardetuned dipole traps, Phys. Rev. A 57: R4106 (1998).

[91] G. Delannoy, S. G. Murdoch, V. Boyer, V. Josse, P. Bouyer, and A. Aspect, Understanding the production of dual Bose-Einstein condensation with sympathetic cooling, Phys. Rev. A 63: 051602 (2001).

[92] M. Mudrich, S. Kraft, K. Singer, R. Grimm, A. Mosk, and M. Weidemüller, Sympathetic cooling with two atomic species in an optical trap, Phys. Rev. Lett. 88: 253001 (2002).

[93] K. Honda, Y. Tasaku, T. Kuwamoto, M. Kumakura, Y. Takahashi, and T. Yabuzaki, Optical dipole force trapping of a fermion-boson mixture of ytterbium isotopes, Phys. Rev. A 66: 021401(R) (2002).

[94] Y. Takasu, K. Honda, K. Komori, T. Kuwamoto, M. Kumakura, Y. Takahashi, and T. Yabuzaki, High-density trapping of cold ytterbium atoms by an optical dipole force, Phys. Rev. Lett. 90: 023003 (2003).

[95] Y. Tasaku, K. Maki, K. Komori, T. Tamako, K. Honda, M. Kumakura, T. Yabuzaki, and Y. Takahashi, Spin-singlet Bose-Einstein condensation of two-electron atoms, Phys. Rev. Lett. 91: 040404 (2003).

[96] S. Jochim, M. Bartestein, A. Altmeyer, G. Hendl, C. Chin, J. Hecker Denschlag, and R. Grimm, Phys. Rev. Lett. 91: 240402 (2003).

[97] E. Timmermans, Degenerate Fermion gas heating by hole creation, Phys. Rev. Lett. 87: 240403 (2001). This interesting mechanism is based on scattering between atoms in the Fermi sea and the energetic background atoms due to the residual pressure in the trapping region, as a sort of Auger effect on the trapped Fermi gas. The requirements for the residual background pressure $\left(<10^{-11}\right.$ Torr $)$ to make this heating source negligible are already well fulfilled by several ultracold atom experimental apparatuses. However, the mechanism can severely limit sympathetic cooling of Fermi atoms due to the collisions experienced with the Bose atoms.

[98] I. Shvarchuck, Ch. Buggle, D. S. Petrov, K. Dieckmann, M. Zielonkowski, M. Kemmann, T. Tiecke, W. von Klitzing, G. V. Shlyapnikov, and J. T. M. Walraven, Bose-Einstein condensation into nonequilibrium states studied by condensate focusing, Phys. Rev. Lett. 89: 270404 (2002).

[99] L. D. Carr, T. Bourdel, and Y. Castin, Limits of sympathetic cooling of fermions by bosons due to particle losses, preprint arXiv:cond-mat/0305441 (19 May 2003). 
[100] R. Napolitano, J. De Luca, V. S. Bagnato, and G. C. Marques, Effect of a finite number of particles in the Bose-Einstein condensation of a trapped gas, Phys. Rev. A 55: 3954 (1997). 